\title{
Association between meteor showers and asteroids using multivariate criteria
}

\author{
B. A. Dumitru ${ }^{1,2,3,4}$, M. Birlan ${ }^{1,3}$, M. Popescu ${ }^{3,1}$, and D. A. Nedelcu ${ }^{3,1}$ \\ ${ }^{1}$ IMCCE, Paris Observatory, 77 avenue Denfert-Rochereau, 75014 Paris Cedex, France \\ e-mail: bogdan.dumitru@obspm. fr \\ 2 Institute of Space Science (ISS), 409, Atomistilor Street, 077125 Magurele, Ilfov, Romania \\ 3 Astronomical Institute of Romanian Academy, 5, Cutitul de Argint Street, 040557 Bucharest, Romania \\ ${ }^{4}$ Faculty of Physics, Bucharest University, 405, Atomistilor Street, 077125 Magurele, Ilfov, Romania
}

Received 17 March 2017 / Accepted 22 July 2017

\begin{abstract}
Context. Meteoroid streams are fragments of matter produced by comets or asteroids which intersects the orbit of Earth. Meteor showers are produced when Earth intersects these streams of matter. The discoveries of active asteroids and extinct comets open a new view of the relation between these objects as possible parent bodies at the origin of meteor showers.

Aims. The aim of this work is to identify the asteroids that can produce or re-populate meteoroid streams by determining the similarity of their orbits and orbital evolution over $10000 \mathrm{yr}$.

Methods. The identification was carried out by evaluating several well known $D$-criteria metrics, the orbits being taken from the IAU Meteor Data Center database and from IAU Minor Planet Center. Finally, we analyzed the physical properties and the orbital stability (in the Lyapunov time sense) of the candidates as well as their possible relationship with meteorites.

Results. 206 near-Earth asteroids (NEAs) were associated as possible parent bodies with 28 meteor showers, according to at least two of the criterion used. 50 of them satisfied all the criteria. Notable finds are: binary asteroid 2000UG11 associated with Andromedids (AND), while the tumbling asteroid (4179)Toutatis could be associated with October Capricornids (OCC). Other possible good candidates are 2004TG10, 2008EY5, 2010CF55, 2010TU149 and 2014OY1. These objects have low albedo, therefore can be primitive objects. Asteroid 2007LW19 which is a fast rotator and most probably has monolithic structure and so its physical characteristic does not support the association found based on the dynamical criteria.
\end{abstract}

Key words. meteorites, meteors, meteoroids - minor planets, asteroids: general

\section{Introduction}

It is now generally accepted that comets can produce meteoroid stream that can be observed on Earth as meteor showers as suggested by Daniel Kirkwood in 1861. Schiaparelli (1867) identified the first pairing of the Perseid meteor shower and comet 109P/Swift-Tuttlle, and Weiss (1868) predicted a large storm of Andromedids meteor shower associated to the comet 3D/Biela. This prediction was confirmed in 1872 and the relationship comet-meteor shower was largely accepted (for an account of these early developments, see Williams 2011).

Clube \& Napier (1984) pointed out that several asteroids had similar orbits to the Taurid stream. Since then, many more associations have been established, based on orbital similarity (Porubčan et al. 2004). Physical studies of asteroids also reinforced this view that asteroids could be associated with meteoroid streams. For example, Weissman et al. (1989) showed that an asteroid could be an extinct comet while Jewitt et al. (2015) found evidence of activity on several asteroids.

When an asteroid revolves around the Sun, it could leave fragments of matter behind it. The processes which produce these fragments are: ejection and disintegration at impacts, rotational instabilities, electrostatic repulsion, radiation pressure, dehydration stresses and thermal fracture, and sublimation of ice (Jewitt et al. 2015). The fragments are called meteoroids, and are in the size range $10 \mu \mathrm{m}$ to $1 \mathrm{~m}$ (Rubin \& Grossman 2010).
It is becoming evident that there is a direct relation between asteroids and meteor showers (see Jopek \& Williams 2013, and all references herein). The Quadrantides meteor shower is associated with the comet $\mathrm{C} / 1490 \mathrm{Y} 1$ and the asteroid 2003EH1 (Jenniskens 2004; Williams et al. 2004; Micheli et al. 2008). The Geminids meteor shower is associated with asteroid (3200)Phaethon (Whipple 1983). For the Taurids Complex the parent body is P/Encke (Whipple \& El-Din Hamid 1952; Wiegert et al. 2009). Several other parent bodies were also identified among the asteroids: 2003UV11, 2003WP21, 2004TG1, and 2005TF50 (Porubčan et al. 2006; Napier 2010; Jopek et al. 2010).

Meteoroids, asteroids, and comets have been permanently interacting with Earth during its existence. In the Earth Impactors Database ${ }^{1}$ there are 190 confirmed impact structures. The biggest cataclysmic event, that highly influences life on Earth, is considered the impact of an asteroid in Chicxulub, Mexico. This took place about 65.5 million years ago (Smit 1980; Alvarez et al. 1980). The crater produced was between 180-200 km in diameter (Hildebrand et al. 1991) and a huge amount of carbon and sulfur was released into the atmosphere. On a long term this new atmospheric composition produced extended darkness, global cooling and acid rain (Toon et al. 1997; Pierazzo et al. 2003). Another recent event is the Tunguska event

1 http://WWW $\cdot$ passc.net/EarthImpactDatabase/ 
in 1908 during which an explosion estimated at 10 to 15 megatons of trinitrotoluene (TNT), occurred near Podkamennaya Tunguska river. The cause of the event was supposed to be the impact of a comet (Shapley 1930) or an asteroid (Kulik 1938). Other studies conclude that there is a high probability that at the origin of the Tunguska event was an asteroid (Farinella et al. 2001). More recently the Chelyabinsk event in Russia, in February 2013 was associated with the impact of an asteroid estimated between 17 to $20 \mathrm{~m}$ in size. The estimated energy of this event was around 440 kilotons of TNT and the shockwave caused 1100 injuries (Popova et al. 2013).

The aim of this paper is to identify the asteroids that can produce or feed the meteor showers. The investigation has been performed considering both the dynamical and physical properties.

\section{Clustering based on dynamical parameters}

All objects in the solar system (planets, asteroids, comets, etc.) are characterized by their its orbital elements: semi-major axis, eccentricity, inclination, longitude of the ascending node, perihelion distance.

The objects that produce meteor showers are identified based on orbit similarities. The most common method of measuring the degree of similarity between the orbits is the so called discrimination criteria or $D$-criterion. This is a distance defined in the orbital elements space. Depending on the authors, the number of parameters and their weights differ: $n=3$ in the case of Asher et al. (1993) and $n=5$ in case of Southworth \& Hawkins (1963) and Jopek (1993). A threshold value $D_{\mathrm{c}}$ is defined, and if $D(X, Y)<D_{\text {c }}$ the orbits are similar and the comet or the asteroid can be associated with the meteor shower.

The first metric using this approach was introduced by Southworth \& Hawkins (1963). Several other metrics were defined by Drummond (1981), Jopek (1993), Asher et al. (1993), Valsecchi et al. (1999), Nesvorný \& Vokrouhlický (2006), Jopek et al. (2008).

In this paper we use the metrics $D_{\mathrm{SH}}$ (Southworth \& Hawkins 1963), $D_{\mathrm{H}}$ (Jopek 1993), and $D_{\mathrm{ACS}}$ (Asher et al. 1993). We briefly introduce these metrics.

$D$-criteria introduced by Southworth \& Hawkins (1963; $D_{\mathrm{SH}}$ ) has the mathematical expression:

$$
\begin{aligned}
{\left[D_{\mathrm{SH}}\right]^{2}=} & \left(q_{X}-q_{Y}\right)^{2}+\left(e_{X}-e_{Y}\right)^{2}+\left(2 \sin \frac{I_{X Y}}{2}\right)^{2} \\
& +\left(\frac{e_{X}+e_{Y}}{2}\right)^{2} \times\left(2 \sin \frac{\Pi_{X Y}}{2}\right)^{2}
\end{aligned}
$$

where $I_{X Y}$ is the angle between the planes of the orbits given by:

$$
I_{X Y}=\arccos \left[\cos i_{X} \times \cos i_{Y}+\sin i_{X} \times \sin i_{Y} \times \cos \left(\Omega_{X}-\Omega_{Y}\right)\right]
$$

and $\Pi_{X Y}$ is the angle between perihelion points:

$$
\begin{aligned}
\Pi_{X Y}= & \omega_{X}-\omega_{Y} \\
& +2 \Gamma \times \arcsin \left(\cos \frac{i_{X}-i_{Y}}{2} \times \sin \frac{\Omega_{X}-\Omega_{Y}}{2} \times \sec \frac{I_{X Y}}{2}\right)
\end{aligned}
$$

where $\Gamma$ is defined by:

$\Gamma=-1 \Rightarrow\left|\Omega_{X}-\Omega_{Y}\right|>180^{\circ}$

$\Gamma=+1 \Rightarrow\left|\Omega_{X}-\Omega_{Y}\right| \leq 180^{\circ}$.

The notations used in Eq. (1) to define the orbital elements are: $q$ (perihelion distance), $e$ (eccentricity), $i$ (inclination), $\Omega$ (longitude of the ascending node), $\omega$ (argument of perihelion).
Jopek (1993) studied the methods introduced by Southworth \& Hawkins (1963) and Drummond (1981) and concluded that they depend mostly on the orbital elements $q$ (in the case of criterion $D_{\mathrm{SH}}$ ) and $e$ (in the case of criterion proposed by Drummond). Based on the above, he proposed a new criterion, $D_{\mathrm{H}}$, defined by:

$$
\begin{aligned}
{\left[D_{\mathrm{H}}\right]^{2}=} & \left(e_{X}-e_{Y}\right)^{2}+\left(\frac{q_{X}-q_{Y}}{q_{X}+q_{Y}}\right)^{2}+\left(2 \sin \frac{I_{X Y}}{2}\right)^{2} \\
& +\left(\frac{e_{X}+e_{Y}}{2}\right)^{2} \times\left(2 \sin \frac{\Pi_{X Y}}{2}\right)^{2} .
\end{aligned}
$$

Finally, as the orbital elements $\omega$ and $\Omega$ evolve rapidly with time, Asher et al. (1993) used only three orbital elements and produce a iterion defined as:

$\left[D_{\mathrm{ACS}}\right]^{2}=\left(\frac{a_{X}-a_{Y}}{3}\right)^{2}+\left(e_{X}-e_{Y}\right)^{2}+\left(2 \sin \frac{i_{X}-i_{Y}}{2}\right)$

where $a$ is the semi-major axis.

\section{Analysis procedures}

We used the orbital elements provided by IAU Minor Planet Data Center $^{2}$ - MPCORB.DAT file. The version from February 2017 contains the data for 728679 objects. The orbital elements of meteor showers were obtained from IAU Meteor Data Center ${ }^{3}$ (Jopek \& Kaňuchová 2014; Jopek \& Jenniskens 2011). This file contains 112 established meteor showers (last updated 25 June 2016 by Z. Kanuchova and T. J. Jopek).

From the databases of meteor showers and asteroids we chose seven fields (name + six orbital elements) that were useful for our statistics. A C ++ code was developed in order to use $D$-criteria metrics from Eqs. (1), (4), and (5). The results consist of associated asteroids designation, their orbital elements, the associated meteor shower, the orbital elements of the radiant and the calculated distance for all metrics $\left(D_{\mathrm{SH}}, D_{\mathrm{H}}\right.$ and $\left.D_{\mathrm{ACS}}\right)$.

\subsection{Choosing a threshold value}

In order to obtain the possible parents $(Y)$ of a meteor shower $(X)$ with the help of the $D$-criteria metrics listed above, we need to establish a limit value $\left(D_{\mathrm{C}}\right)$. If the distance $D(X, Y)$ between $X$ and $Y$ is smaller than $D_{\mathrm{C}}$, that object has a possibility of being a parent. If $D(X, Y)>D_{\mathrm{C}}$, the object will be ignored.

We found in the literature that there are different methods for setting the threshold. In Asher et al. (1993) the authors calculated the associations for Taurids Complex (TC) using $D_{\text {ACS }}$ metric with a threshold of 0.26 and obtained 25 associated asteroids (see Asher et al. 1993, Table 1). They suggested that a threshold of 0.2 corresponds to the minimum probability for the TC asteroids. In Porubčan et al. (2006) the authors use $D_{\mathrm{SH}}$ metric with a threshold set to 0.3 to determinate the associations for TC from 3380 NEOs and they obtained 91 associated objects. In a second step a computation backward in time for 5000 years allows nine associated NEOs to remain. Rudawska et al. (2012a) tests $D$-criterion metrics, $D_{\mathrm{SH}}$ and $D_{\mathrm{H}}$, using the model of generation and evolution of meteoroid stream in the solar system from Vaubaillon et al. (2005). They determined a threshold of 0.084 for $D_{\mathrm{SH}}$ and 0.077 for $D_{\mathrm{H}}$, when a meteor is associated to the meteoroid stream. In Šegon et al. (2014), authors use metrics $D_{\mathrm{SH}}$

\footnotetext{
2 http://wWw . minorplanetcenter.net

3 http://WWW.astro.amu.edu.pl/ jopek/MDC2007
} 
and $D_{\mathrm{H}}$ with cutoff values set to 0.15 to find asteroids associated with meteor showers. They found 43 associated asteroids with inclination $>15^{\circ}$ that can be associated to streams containing ten or more meteor orbits. The cutoff values for this paper were selected following Lindblad (1971a,b), and Jenniskens (2006).

Ryabova (2016) use metrics $D_{\mathrm{SH}}$ to evaluate the dispersion of a Geminid stream model composed by a sample with three different meteoroid masses. The integration of orbits backward in time for 2000 years show that for this population the $D_{\mathrm{SH}}$ do not exceed 0.2. Also, this is less than 0.046 when it is estimated for the meteor shower (Ryabova 2016).

The number of associated asteroids is highly dependent on the selected threshold. Each metric requires a different threshold, because the method of computation will produce different statistical distances (see Jopek 1993, Table 1).

Our analysis used a new algorithm in two steps for defining a threshold value.

Firstly, we ran the metrics for different values, between 0 to 0.5 for several values of $D_{\mathrm{C}}$. From these runs we selected the number of associations and the meteor showers that have associated objects. Then we defined a global parameter which could characterize all the meteor showers (Eq. (6)).

$A V_{D_{\mathrm{C}}}=\frac{N_{D_{\mathrm{C}}}}{M_{D_{\mathrm{C}}}}$

where $N_{D_{\mathrm{C}}}$ is the total number of associated asteroids for a metric, and $M_{D_{\mathrm{C}}}$ is the number of meteor showers that could be produced by these asteroids. $A V_{D_{\mathrm{C}}}$ is the average number of associated asteroids per meteor shower and is dimensionless with no physical meaning. It represents just a way to qualify the evolution of the clustering.

This average dimensionless parameter was used further for the definition of the threshold value (Fig. 1).

An important change in slope occurs for $A V=18$ (Fig. 1a). This value will be used for finding the threshold value (Fig. 1b). Thus, our findings for the cutoff values are: $D_{\mathrm{ACS}}=0.06, D_{\mathrm{SH}}=$ 0.21 , and $D_{\mathrm{H}}=0.19$.

\subsection{Excluding from the threshold the D-parameter uncertainty}

The second phase is to eliminate from the associated asteroid, those objects with large uncertainty. For this we take into consideration the standard deviation for each orbital element (NEODYS $^{4}$ database, version of February 2017).

To obtain the uncertainty for the $D$-parameter, we propagate the standard deviation for each asteroid orbital element through the metrics. The function used to determine the uncertainty for $D$-parameter is:

$$
\sigma_{D}=\sqrt{\left(\frac{\partial f}{\partial x}\right)^{2} \times\left(\sigma_{x}\right)^{2}+\left(\frac{\partial f}{\partial y}\right)^{2} \times\left(\sigma_{y}\right)^{2}+\left(\frac{\partial f}{\partial z}\right)^{2} \times\left(\sigma_{z}\right)^{2}+\ldots}
$$

where $\frac{\partial f}{\partial x}, \frac{\partial f}{\partial y}, \frac{\partial f}{\partial z}$, etc. is the partial derivatives of the metric for each orbital element and $\sigma_{x, y, z}$ is the standard deviation of each orbital element.

The asteroid should be associated if:

$D c>D+3 * \sigma_{D}$

\footnotetext{
4 http://newton.dm.unipi.it/neodys/
}

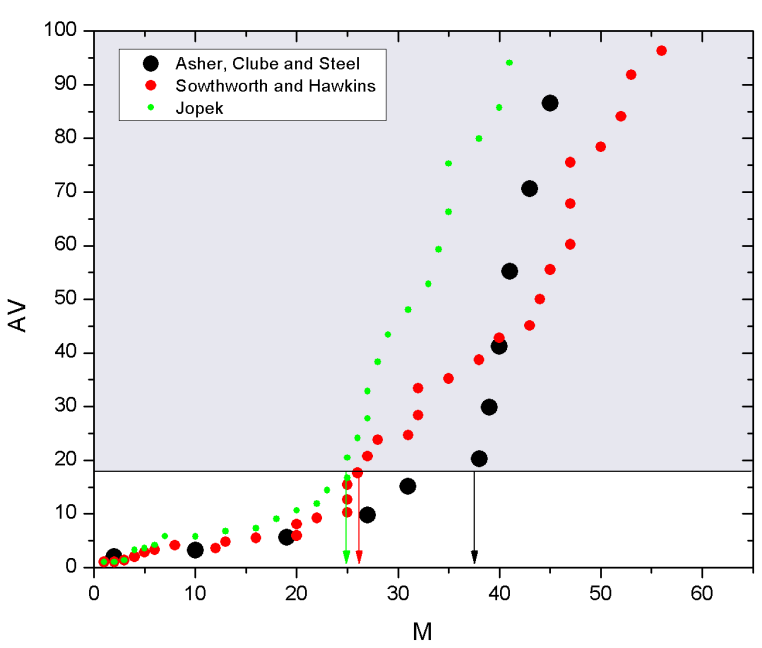

(a)

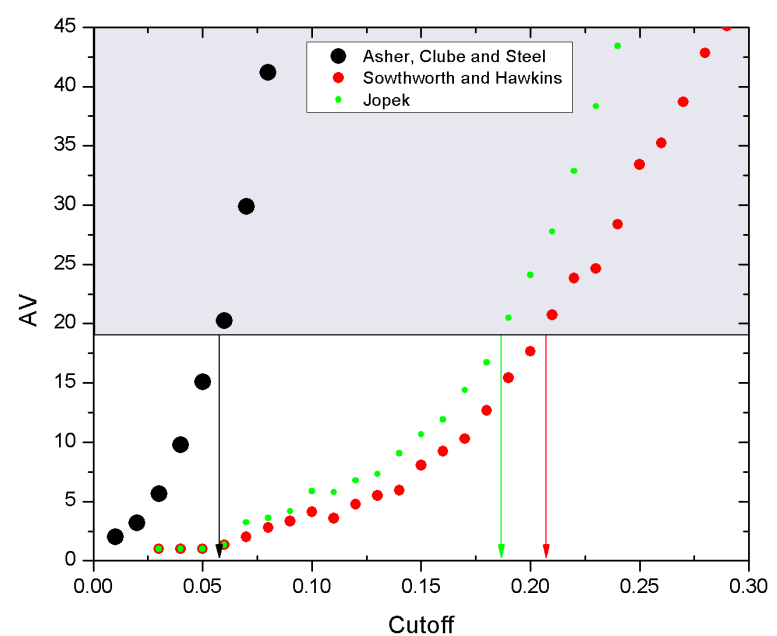

(b)

Fig. 1. Threshold selection. Panel $a$ : representation of the average number of associated asteroids per meteor shower (AV) versus the number of meteor showers (M) that could be produced by the asteroids. Panel $b$ : representation of average number of associated asteroids per meteor shower (AV) versus cutoff value.

where $D c$ is the threshold, $D$ is the $D$-parameter for the asteroid and $\sigma_{D}$ is the computed uncertainty for $D$-parameter through error propagation presented in Eq. (7).

\subsection{Filtration: objects with high, medium and low probability}

In our paper we consider, parent body objects with high probability those asteroids which were associated to a meteor shower by all three metrics. The medium probability will be considered for the asteroids which were associated by two metrics, while the low probability represents the amount of asteroids associated to a meteor shower just by one metric. By using several metrics for our statistics, it seems more appropriate to consider several clustering approaches giving similar results. Thus, we will concentrate our analysis to the possible parent bodies, classified by us, as high and medium probability.

Corvids (COR) and h Virginids (HVI) have 54\% of the total associated asteroids. For COR and HVI we decided to take into consideration only the high probability objects. 


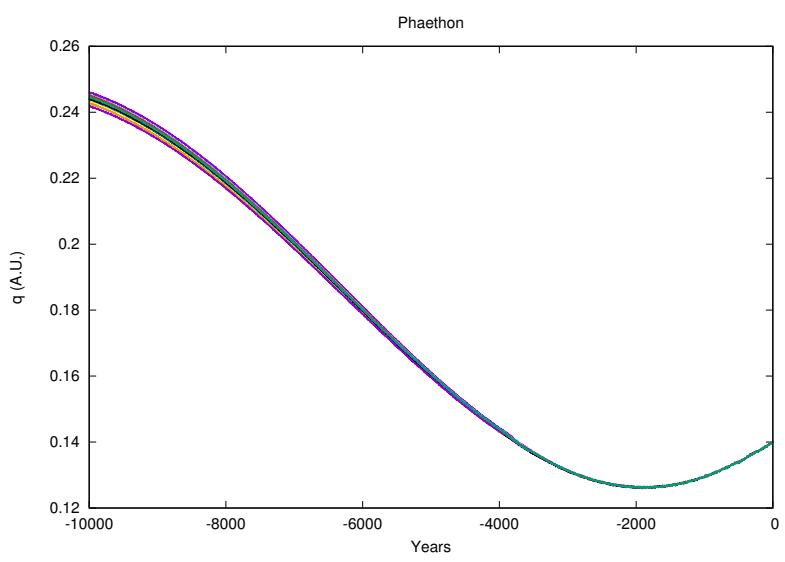

(a)

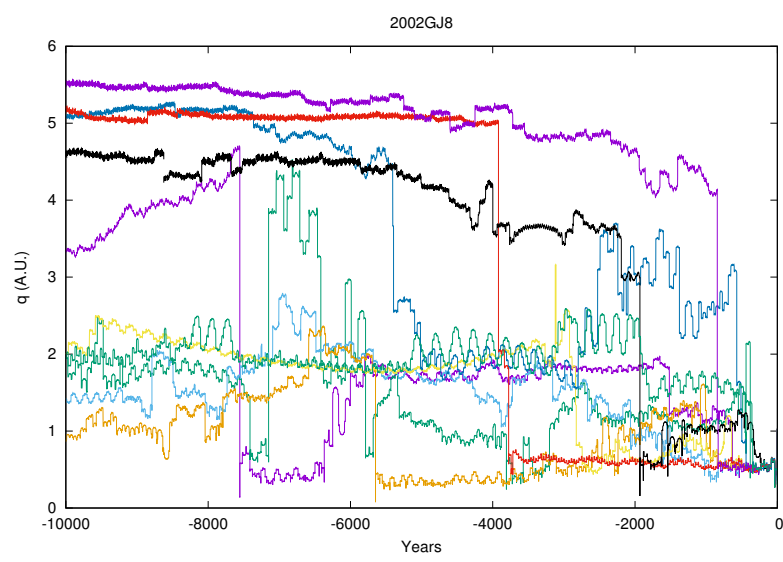

(b)

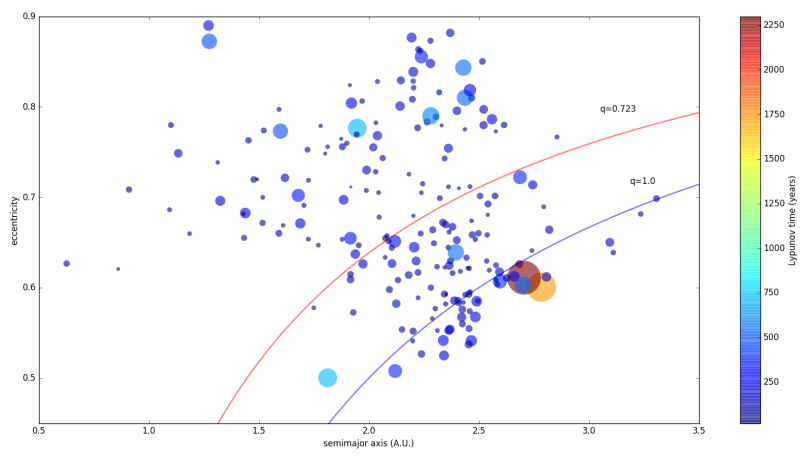

(c)

Fig. 2. Asteroids orbital stability according to our criteria of stable (panel a) or unstable (panel $b$ ) orbit and the Lyapunov time (panel c). In panel $a$ we represented the asteroid (3200)Phaethon's perihelion evolution. This asteroid has a stable orbit $\left(T_{\mathrm{L}}=226 \mathrm{yr}\right)$. In panel $b$ we represent the asteroid 2002GJ8 perihelion evolution. This asteroid has an unstable orbit $\left(T_{\mathrm{L}}=63 \mathrm{yr}\right.$ ). And in panel $c$ is the representation of $T_{\mathrm{L}}$ for all associated asteroids.

\subsection{Additional filtration: orbital evolution and Lyapunov time}

A supplementary investigation was done for the determination of orbital stability of asteroids using clones (Nedelcu et al. 2014).

For each asteroid we generated ten clones using a Gaussian distribution in the space of the six orbital elements with the corresponding standard deviation:

$\sigma_{\text {clones }}=3 \times \sigma_{\text {asteroid }}$

with $\sigma_{\text {asteroid }}$ for each element obtained from NEODYS service (Chesley \& Milani 1999). For each asteroid the clones were integrated backward in time for $10000 \mathrm{yr}$ using a realistic dynamical model of the solar system described by Nedelcu et al. (2010) modified to use an 80-bit extended precision data type. Examples of our numerical integration are in Figs. 2a, b.

The orbital dispersion of clones (Appendix D) is due both to the current uncertainty of the orbital elements and to the inherently chaotic nature of NEO dynamics. Backward numerical integrations of meteoroids orbits along with their presumed parent body may be able to identify the epoch of stream formation by finding the intersection of orbits of meteoroids and that of asteroid (Gustafson 1989).

This kind of approach is, however, complicated when one includes the current uncertainties of meteoroids orbital elements (Ryabova et al. 2008). Highly accurate orbits are required in order to infer a reliable stream age using the above method.

For relatively well constrained orbits of the showers, the stochastic nature of NEO orbital evolution is a second limiting factor that has to be considered and it motivates our numerical study (Abedin et al. 2017). A meaningful parent-shower association cannot be determined beyond few Lyapunov times in the past.

This kind of analysis can be reliably employed only for a few Lyapunov times back in time. For this reason we computed the Lyapunov time $\left(T_{\mathrm{L}}\right)$ from the solution of the variational equations that were integrated backward in time together with the equations of motion (Tancredi et al. 2001). We limited this integration run to $2000 \mathrm{yr}$ which is approximately ten times the typical value of $T_{\mathrm{L}}$ for near-Earth asteroids (NEA).

The results are presented in Table E.1 and in Fig. 2c. 85\% of asteroids have a $T_{\mathrm{L}}$ shorter than 200 years, a result in agreement with Tancredi (1998). According to the values of the $T_{\mathrm{L}}$ we propose two distinct categories of NEA:

1. If $T_{\mathrm{L}}>100 \mathrm{yr}$, the asteroid has stable orbit. These asteroids may be long-time contributors to the meteor flux.

2. If $T_{\mathrm{L}}<100 \mathrm{yr}$, the asteroid has unstable orbit. These asteroids may be the current contributors to the supply of that meteor shower.

\section{Results}

In summary, considering those thresholds and after all the filters used, our analysis contains 50 asteroids with high probability and 173 with medium probability. We find multiple occurrences (Table 1) for several asteroids (i.e., 2012KA4, 2004TG10, 2003UV11, 2007UL12, etc.). 
B. A. Dumitru et al.: Association between meteor showers and asteroids using multivariate criteria

Table 1. Set of 28 meteor showers and their associated asteroids ( 50 with high probability and 173 with medium probability).

\begin{tabular}{|c|c|c|c|}
\hline Cod Name & High probability & Medium probability & Parent body \\
\hline AND Andromedids & 0 & $\begin{array}{l}\text { (152770)1999RR28, 2000UG11, (267729)2003FC5, 2003UQ25, } \\
\text { 2004GB2, 2009ST103, 2009TA1, 2009WJ1, 2010TV54, 2012FG, } \\
\text { 2012TT231, 2012VB5, 2016FC14, 2016TW18, 2016UP36, } \\
\text { 2016VQ }\end{array}$ & 3D/Biela \\
\hline APS Daytime April Piscids & 0 & (438105)2005GO22, 2012KA4 & 2005NZ6 \\
\hline AUD August Draconids & 0 & 2002GJ8, 2016NO16 & - \\
\hline BHY $\beta$ Hydrusids & 0 & 2016RX19 & - \\
\hline BTA Daytime $\beta$ Taurids & 0 & $\begin{array}{l}\text { 2003UV11, 2004TG10, 2007UL12, 2010TU149, 2011TC4, } \\
\text { 2011TX8, 2012UR158, 2014NK52, 2015TX24, 2015VH66, } \\
\text { 2016TP18 }\end{array}$ & 2P/Encke, 2004TG10 \\
\hline CAP $\alpha$ Capricornids & 2008BO16, 2015DA54 & $\begin{array}{l}\text { 2001EC, 2002CB26, 2002NW, 2011CG50, 2012BL14, } \\
\text { 2012CC29, 2014OO6, 2015CE1, 2015CP, 2015DE54, 2015NJ3, } \\
\text { 2016BN14, 2016BP14, 2016BV14, 2016CL137, 2016CW264 }\end{array}$ & 169P/NEAT \\
\hline DSX Daytime Sextantids & 0 & $(155140) 2005 \mathrm{UD}$ & (155140)2005UD \\
\hline EVI $\eta$ Virginids & 2003FB5, 2006UF17 & $\begin{array}{l}\text { (455176)1999VF22, 2000DO1, 2002CN15, } 2007 \mathrm{EJ} 88, \\
\text { 2008CA22, 2008VL14, 2010CF55, 2010TN55, 2015ER, } \\
\text { 2015FD35, 2016ES155, 2016EV28 }\end{array}$ & $\mathrm{D} / 1766 \mathrm{G} 1$ \\
\hline GEM Geminids & (3200)Phaethon & 0 & (3200)Phaethon \\
\hline KCG $\kappa$ Cygnids & 0 & 2016NO16 & - \\
\hline MKA Daytime $\kappa$ Aquariids & $(430544) 2002 \mathrm{GM} 2$ & 2011FZ22, 2013NE19 & 2002 EV11 \\
\hline NCC Northern $\delta$ Cancrids & $(85182) 1991 \mathrm{AQ}$ & 2013YL2, 2015PU228 & $(85182) 1991 \mathrm{AQ}$ \\
\hline NIA Northern $\iota$ Aquariids & 0 & 2006PF1, 2012KA4, 2015РM307 & - \\
\hline NTA Northern Taurids & $\begin{array}{l}\text { 2004TG10, 2010TU149, } \\
\text { 2012UR158, 2014NK52 }\end{array}$ & 2001UX4, 2003UV11, 2011TC4, 2016VK & 2P/Encke \\
\hline OCC October Capricornids & 0 & $\begin{array}{l}\text { 2002RC117, 2005TD49, 2006OC5, 2006XA3, 2008XU2, } \\
\text { 2009ST171, 2010RB12, 2011OL51, 2011YY62, 2012XM134, } \\
\text { 2013WX44, 2013XT21, 2014XE32, 2014XL6, 2015TL143, } \\
\text { 2016PN38, 2016RZ40, 2016WN48, (4179)Toutatis }\end{array}$ & $\mathrm{D} / 1978 \mathrm{R} 1$ \\
\hline
\end{tabular}

\begin{tabular}{lllr}
\hline OER $o$ Eridanids & 0 & 2015KK & \\
\hline ORS Southern $\chi$ Orionids & 0 & 2007UL12, 2013CT36, (2101)Adonis & 2010LU108, 2002XM35 \\
\hline PPU $\pi$ Puppids & 2011TA4 & (417634)2006XG1, 2008RT, 2008XQ2, 2010UY6, 2015VR65, & 26P/Grigg-Skjellerup \\
& & & \\
\hline SCC Southern $\delta$ Cancrids & 0 & 2016TJ18 & 2001YB5 \\
\hline SMA Southern Daytime May Arietids 2001QJ96 & 2012KA4, 2016LW9 & - \\
\hline SSG Southern $\mu$ Sagittariids & 2011BM45, 2016CM246 & 1998LE, 1999LW1, 2002AU5, 2007YP56, 2011BW10, & - \\
& & 2011BY18, 2011CT4, 2012BJ14, 2012BU61, 2013AB65, & \\
& & 2015BA513, 2015MN11, 2016CA136, 2016NG22 & \\
\hline
\end{tabular}

\begin{tabular}{llcl}
\hline STA Southern Taurids & 2007UL12, & 2012ES10, 2003UV11, 2005TB15, 2007RU17, 2010TU149, 2011TC4, & 2016CM246
\end{tabular}

\begin{tabular}{|c|c|c|c|}
\hline TAH $\tau$ Herculids & 0 & $\begin{array}{l}\text { (455299)2002EL6, 2003LW1, 2004HC39, 2005JJ91, 2006HQ30, } \\
\text { 2009FU4, 2010GH65, (436671)2011SV71, 2013JT17, 2014OY1, } \\
\text { 2016HN3, (3671)Dionysus }\end{array}$ & 73P/Schwassmann-Wachmann 3 \\
\hline
\end{tabular}

\begin{tabular}{lllr}
\hline XSA Daytime $\xi$ Sagittariids & 0 & $(325102) 2008 \mathrm{EY} 5,2015 \mathrm{FQ} 117$ & - \\
\hline ZPE Daytime $\zeta$ Perseids & $(162195) 1999$ RK45 & 0 & 2P/Encke \\
\hline AVB $\alpha$ Virginids & 2002FU5 & 1997GD32, 1998SH2, (446791)1998SJ70, 2001TA2, 2004SA20, & 1998SH2
\end{tabular}
2004VY14, 2005RW3, 2005TE, 2006JO, 2007GU1, 2009HS44, 2009SB15, 2010FL, 2010GE35, 2011EF17, 2011GP65, 2011HP4, 2011TJ, 2012FQ62, 2012JU, 2012LJ, 2012TT5, 2014HT178, 2014HU2, 2014MC6, 2014XD32, 2015FQ, 2015GJ13, 2016JD18, 2016JS5, 2016RO40, 2016SF

COR Corvids 1996MQ, (162058)1997AE12, 1999RK33, 2002PX39, 2003RE2, 2004MC, 2004RW10, 2005MR1, 2005OF3, 2007LW19, 2009QG2, 2009SX17, 2011QE38, 2012KZ41, 2012LT, 2013PW31, 2013RN21, 2013RU9, 2014KH39, 2014OM207, 2016LE10, 2016LZ8, 2016MS, 2016PD40, 2016QY1

HVI h Virginids 2001SZ269, 2010RL43, 2010TP55, 2014HU2, 2014JH15

Notes. The asteroids in bold are associated to several meteor showers. Parent body column is the associated parent body from IAU Meteor Data Center as of 25 June 2016. Corvids (COR) and h Virginids (HVI) are on the last two lines and only high probability associations are presented. 


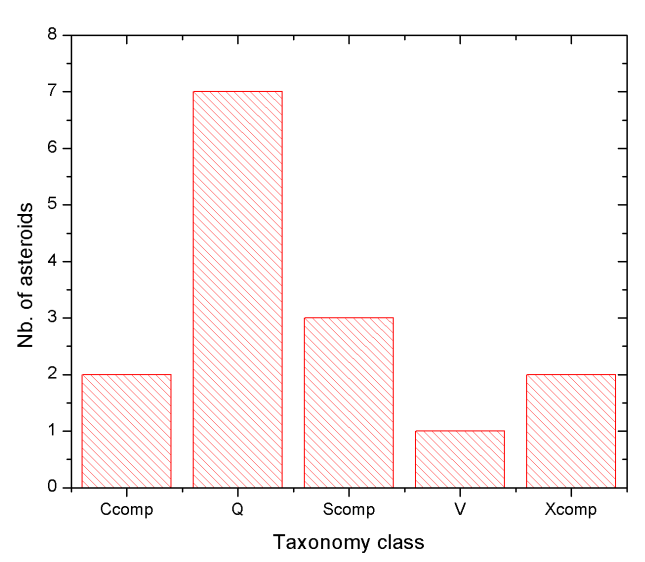

(a)

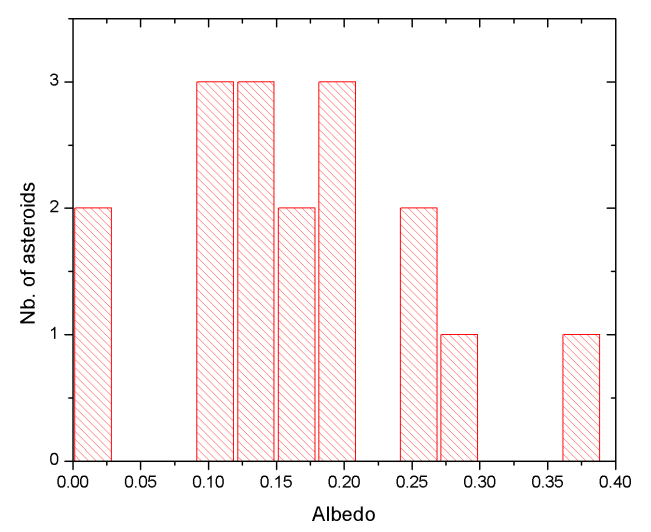

(c)

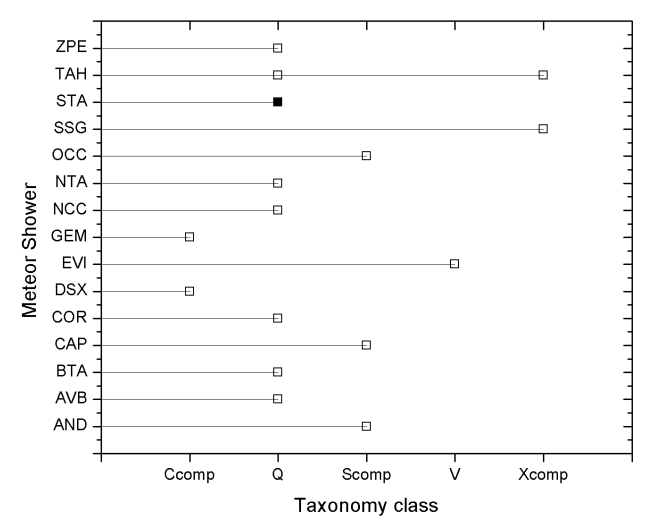

(b)

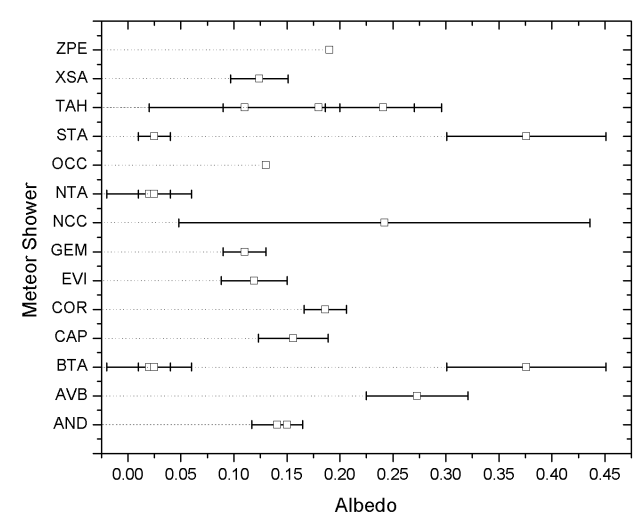

(d)

Fig. 3. Physical data found in EARN database about the associated asteroids. For taxonomic classes of our sample, we present the number for asteroids with a taxonomic class (panel $a$ ) and the taxonomic class associated for each meteor shower (panel $b$ ). For the albedo find from our sample, we present the number of asteroids with albedo (panel c) and which albedo was associated to each meteor shower (panel d). For three objects we lack the error bar. In graphs, the empty squares represents one object and full squares represents two objects.

\subsection{Dynamical view}

As expected, all asteroids selected by our analysis belong to NEAs population. From the total number of associations asteroids $82.6 \%$ have Apollo type orbits, thus passing Earth orbit, and $26 \%$ are classified as potential hazardous asteroids (PHA).

For every meteor shower we compared our results with IAU Meteor Data Center for known associated parent bodies (the Parent body column in Table 1). Knowing the parent bodies of the meteor showers we can conclude:

1. We find the same associations with high and medium probability between (3200)Phaethon and Geminids (GEM; Whipple 1983), (155140)2005UD and Daytime Sextantids (DSX; Ohtsuka et al. 2005), (85182)1991AQ and Northern $\delta$ Cancrids (NCC; IAU Meteor Data Center), 2004TG10 and Taurid Complex (BTA and NTA; Jenniskens 2006), 2010 TU149 and Taurid Complex (Rudawska et al. 2012b), 1998SH2 and $\alpha$ Virginids (AVB; IAU Meteor Data Center), (374038)2004HW and Corvids (COR; IAU Meteor Data Center) and (196256)2003EH1 and Quadrantids (QUA; Jenniskens 2004; Williams et al. 2004; Micheli et al. 2008).

2. We find the same associations with low probability between 2001YB5 and Southern $\delta$ Cancrids (SCC; Meng et al. 2004), 2005NZ6 and Daytime April Piscids (APS; IAU Meteor Data Center) and 2010LU108 and Southern $\chi$ Orionids (ORS; IAU Meteor Data Center).
3. We do not obtain the association between 2002EV11 and Daytime $\kappa$ Aquariids (MKA; IAU Meteor Data Center).

\subsection{Physical parameter view}

A systematic search for physical parameters of objects in Table 1 was performed using the European Asteroid Research Node (EARN) database ${ }^{5}$ (version February 2017), NEOWISE: the Wide-field Infrared Survey Explorer (WISE) database $^{6}$ (Mainzer et al. 2016) and Asteroids Lightcurve Photometry Database ${ }^{7}$ (version February 2017). From our sample of 206 NEAs, 15 asteroids have spectra in the visible and nearinfrared, 15 asteroids have an associated taxonomic class, and 17 asteroids have an albedo (Fig. 3).

Asteroid surfaces have specific reflective properties which could be characterized by their spectra. Visible and near-IR spectra helps us to identify chemical and mineralogical properties of asteroid surfaces (Reddy et al. 2015). More spectral data of asteroids are required for a global image of compositional properties which could be derived from reflectance spectra.

In Fig. 3a, the associated asteroids with taxonomic classes are plotted. This plot shows that our sample is largely dominated by objects of the S-complex. Q-types (DeMeo et al. 2009) are

\footnotetext{
5 http://earn.dlr.de

6 https://sbn.psi.edu/pds/resource/neowisediam.html

7 http://alcdef.org/
} 


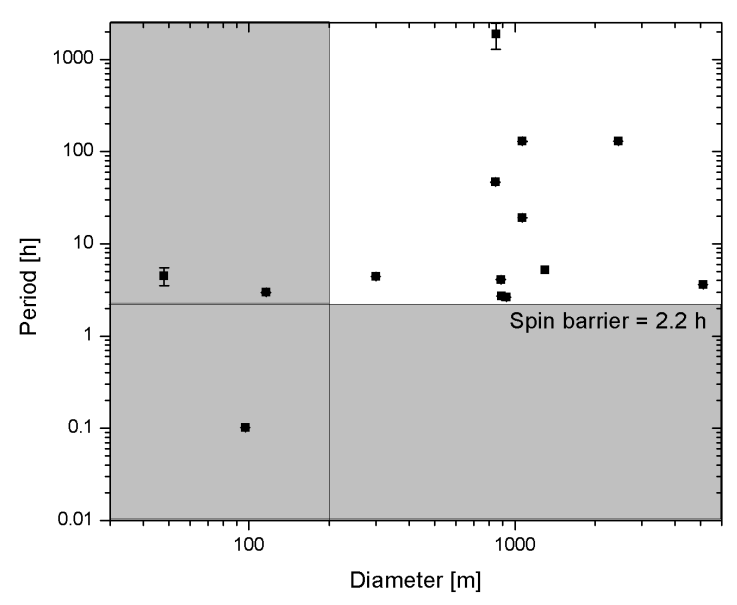

Fig. 4. Rotational periods of associated asteroids from our sample found in the literature. The value of $2.2 \mathrm{~h}$ represents the spin barrier for an asteroid larger than $200 \mathrm{~m}$ (Pravec et al. 2006). In this graph, the white rectangle present our objects of interest.

also largely represented, and their presence is associated with a resurfacing of S-type asteroids during close approach of the Earth (Binzel et al. 2010). Looking at our results and comparing with the statistic of all NEAs we observe a discrepancy between $\mathrm{Q}$ and S-types. The statistic of all NEAs the most predominant taxonomic classes are S-type followed by Q-type, while in our sample it is the inverse. But if we take into account that Q-type are fresh surface S-type asteroids, our result is not surprising. In Fig. $3 \mathrm{~b}$ we present the taxonomic classes of associated asteroids find with taxonomic class vs. meteor showers. If the parent body of a meteor shower is of a certain taxonomic type, the meteor shower needs to have the same taxonomic class. From this we can speculate that two meteor showers have C-type taxonomic class, seven have Q-type, three S-type, one V-type, one X-type and TAH with Q or/and X-type.

In Fig. 3c we present asteroids with known albedo. The asteroids with an albedo smaller than 0.15 (excluding the metallic ones) are akin to primitive objects belonging to taxonomic class such as C, D, B or G (Fulchignoni et al. 2000) while an albedo larger than 0.15 could be associated to objects closer to ordinary chondrites (S-complex), or to objects which experienced partial or total melting ( $\mathrm{V}, \mathrm{O}, \mathrm{A}$, or $\mathrm{X}$ taxonomic classes). Approximately 17 objects from our sample have albedo recorded in the EARN database. In our asteroids sample we can find a balance between high and low albedo, considering the value $\rho_{v}=0.15$ (eight objects exhibits albedos lower than this value, while nine objects have values of albedo larger than 0.15 ). In Fig. $3 \mathrm{~d}$ we can see the associated asteroids albedo values found in literature attributed to each meteor shower.

From the associated asteroids $7.25 \%$ have spectra in the visible and/or near-IR and $8.21 \%$ have known albedo.

We found 14 asteroids from our sample with rotational periods. The histogram of associated asteroids with known rotational periods is presented in Fig. 4.

Pravec et al. (2006) studied the relationship between asteroid dimensions and their rotational periods. They found a limit of this rotational period, and they called this parameter spin barrier. The spin barrier was estimated at about $2.2 \mathrm{~h}$. This spin barrier helps us to distinguish between rubble-piles and monolithic NEAs. In the assumption of a rubble-pile structure, if an asteroid is larger than $200 \mathrm{~m}$, this asteroid needs to have a rotational period value larger than the spin barrier one.
For fast-rotating asteroids (Polishook et al. 2016), to overcome their own centrifugal force, a monolithic structure is required. In the case of a fast rotator, the probability of producing fragments or meteoroids is fairly low.

Asteroids with rubble-pile structures, over $200 \mathrm{~m}$, need to have a small rotation rate because their structure is maintained by their own (low!) self-gravity, or the cohesive forces of bonded aggregates (Richardson et al. 2009).

In the context of these findings, it seems realistic to investigate in detail the asteroids of our sample which could have a rubble-pile structure. Binary asteroids as well as slow-rotators seem to be more appropriate as objects that can be easily desegregate, thus producing meteoroids.

In our data we found one fast-rotating asteroid, namely 2007LW19 that has a rotational period of $0.10169 \pm 0.00014 \mathrm{~h}$ (Kwiatkowski et al. 2010) and diameter ${ }^{8}$ between $60-134 \mathrm{~m}$. More probably this asteroid has monolithic structures, therefore we can only speculate on their ability of producing meteoroids. Its orbit was classified as unstable (see Figs. D.2c). Its association with Corvids (COR) and $\mathrm{h}$ Virginids (HVI) might be also under debate.

Meteor showers are characterized using geocentric entry speed $\left(V_{\mathrm{g}}\right)$ taken from Jenniskens et al. (2016a) and the zenithal hourly rate (ZHR) and maximum activity date from the International Meteor Organization (IMO) website ${ }^{9}$.

The asteroids spectral data were reviewed using the Modeling for Asteroids (M4AST ${ }^{10}$ ) tool. M4AST is an online tool devoted to the analysis and interpretation of visible and near-infrared reflection spectra of asteroids by querying databases containing more than 6000 spectra (Popescu et al. 2012; Birlan et al. 2016).

We will make a short description of meteor showers and the related asteroids with physical data.

Andromedids (AND). This meteor shower has a $V_{\mathrm{g}}=$ $18.2 \mathrm{~km} \mathrm{~s}^{-1}$. In our search we found three asteroids with physical data associated with this meteor shower. (267729)2003FC5 taxonomic class was determined as S-type (Thomas et al. 2014). Using the bridge between M4AST routines and SMASS-MIT UH-IRTF (MINUS database ${ }^{11}$ ) we found one spectrum for this asteroid. Only a part of the spectrum of (267729)2003FC5 is reliable (from 0.8 to $1.5 \mu \mathrm{m}$ ). The feature around $1 \mu \mathrm{m}$ is matched by the K-type (Fig. C.1n).

2009ST103 has a known albedo $0.141 \pm 0.024$ (Mainzer et al. 2016). For 2000UG11 an assumed albedo 0.15 is associated (Binzel et al. 2002).

From radar observations (Margot et al. 2002) concludes that 2000 UG11 is a binary object. More generally, the formation of binary objects (the increase in spin rate due to YORP effect, followed by mass-loss and ended with a satellite in a close orbit) require a rubble-pile or gravitational aggregate structure (Scheeres 2007; Walsh et al. 2012). 2000UG11 is a good candidate as parent body for this shower.

From orbital evolution we observe that asteroids 2003FC5 and 2009ST103 have stable orbits $\left(T_{\mathrm{L}}=119\right.$ respectively 389 , see Figs. D.2f and D.1k), while 2000UG11 has unstable orbit $\left(T_{\mathrm{L}}=85 \mathrm{yr}\right.$, see Fig. D.1f).

$\alpha$ Capricornids $(C A P)$. This meteor shower has a $V_{\mathrm{g}}=$ $23 \mathrm{~km} \mathrm{~s}^{-1}$ and a ZHR $=5$ for the maximum of 30th of July. We

8 Diameter range derived from magnitude and assumed albedo for $\mathrm{C}$ and $S$-type -0.04 and 0.20 respectively.

9 http://www.imo.net/files/meteor-shower/cal2017.pdf

10 http://spectre.imcce. fr/m4ast/index . php/index/home

11 http:smass.mit.edu/minus.html 
found two asteroids with physical data, namely 2001EC which is a Sq-type taxonomic class (Binzel et al. 2004) and 2002NW which has an albedo of $0.156 \pm 0.033$ (Mainzer et al. 2016).

From investigation backward in time for asteroids 2001EC and $2002 \mathrm{NW}$ we obtain unstable orbit for both asteroids $\left(T_{\mathrm{L}}=\right.$ 34 respectively 41 yr, see Figs. D.1g and D.1j).

Daytime Sextantids (DSX). This shower has $V_{\mathrm{g}}=$ $32.9 \mathrm{~km} \mathrm{~s}^{-1}$ and a ZHR $=5$ for the maximum of 27 th of September. We found just one asteroid with physical data, namely 2005UD. We associated this asteroid with medium probability to DSX. The $V_{\mathrm{g}}=32.9 \mathrm{~km} \mathrm{~s}^{-1}$ is quite similar to the one of DSX (Jenniskens et al. 2016a). Ohtsuka et al. (2005) classified this object as a C-type asteroid.

Several authors concluded that 2005UD could be a fragment from the asteroid (3200)Phaethon (Ohtsuka et al. 2006; Jewitt \& Hsieh 2006; Kinoshita et al. 2007; Jones et al. 2016).

Our numerical simulations for 2005UD show that this asteroid have stable orbit $\left(T_{\mathrm{L}}=478 \mathrm{yr}\right.$, see Fig. D.1o).

$\eta$ Virginids (EVI). This meteor shower has a $V_{\mathrm{g}}=$ $26.6 \mathrm{~km} \mathrm{~s}^{-1}$. In our search we found two asteroids with physical data. 2000DO1 was classified as V-type based on spectral data obtained from visible and near-infrared observations up to $1.6 \mu \mathrm{m}$ (Binzel et al. 2004). For the asteroid 2010CF55 only an albedo of $0.119 \pm 0.031$ was found (Mainzer et al. 2014). The available spectrum of 2000DO1 covers the wavelengths between 0.5 and 1.6. The curve matching methods from M4AST indicate a Q-type (mean squared error 0.0077). However, the band at $1 \mu \mathrm{m}$ is not matched by this type. This band is typically for a V-type (Fig. C.1i). Thus, we conclude a V-type classification, although the mean square error is higher (0.016).

The orbital evolution of 2000DO1 and 2010CF55 show that both have unstable orbit $\left(T_{\mathrm{L}}=38\right.$ respectively $65 \mathrm{yr}$, see Figs. D.1e and D.2g).

Geminids (GEM). This meteor shower has a $V_{\mathrm{g}}=$ $33.8 \mathrm{~km} \mathrm{~s}^{-1}$ and a ZHR of 120-130 (Rendtel 2004) for the maximum of 14th of December. Its parent body (3200)Phaethon has a $V_{\mathrm{g}}=33.9 \mathrm{~km} \mathrm{~s}^{-1}$, quite similar to this meteor shower (Jenniskens et al. 2016a). Phaeton was taxonomically classified as a B-type or F-type (Licandro et al. 2007; Fornasier et al. 2006; Binzel et al. 2004).

This asteroid was intensively studied. Even if the parent body of Geminids stream is an asteroid, its structure model agrees with the cometary scenario of its origin, which leads to the conclusion that (3200)Phaethon could be a extinct comet (Ryabova 2007). In 2009 and 2012 it exhibits anomalous brightening around its perihelion, which has been interpreted as the ejection of dust particles (Li \& Jewitt 2013; Jewitt \& Li 2010). Ryabova (2012) modeled this dust ejection and the evolution of the meteoroid swarm and conclude that the approach of the swarm to the Earth will be in 2014, 2017, 2018 and 2020 Statistics made between 2009 and 2015 do not confirm an increase in Geminid activity (Miskotte 2016). An important discovery about this asteroid was a tail detection (Jewitt et al. 2013). Other studies show that (3200)Phaethon has similar orbit with asteroid 2005UD (Ohtsuka et al. 2006; Jewitt \& Hsieh 2006; Kinoshita et al. 2007; Jones et al. 2016), which suggests that (3200)Phaethon and 2005UD might have common origin. This asteroid has a stable orbit $\left(T_{\mathrm{L}}=226 \mathrm{yr}\right.$, see Fig. $\left.2 \mathrm{a}\right)$.

$\delta$ Cancrid Complex. This complex contains Northern $\delta$ Cancrids (NCC) and Southern $\delta$ Cancrids (SCC). The NCC meteor shower has an estimated value of geocentric entry speed of $V_{\mathrm{g}}=27.2 \mathrm{~km} \mathrm{~s}^{-1}$. We found that (85182)1991AQ is associated with high probability (i.e. all three metrics found this object under the threshold) to NCC. Based on ECAS filters (Zellner et al. 1985) this asteroid was classified as Q-type by Wisniewski et al. (1997). NEOWISE estimation of its albedo is $0.242 \pm 0.194$ (Mainzer et al. 2016). (85182)1991AQ has an unstable orbit $\left(T_{\mathrm{L}}=94 \mathrm{yr}\right.$, see Fig. D.1a).

October Capricornids (OCC). This shower has a $V_{\mathrm{g}}=$ $10 \mathrm{~km} \mathrm{~s}^{-1}$. (4179)Toutatis is the only associated asteroid from our sample which has physical data. (4179)Toutatis was classified as S, Sk, or Sq (Davies et al. 2007; Binzel et al. 2004; DeMeo et al. 2014). Its albedo was estimated around 0.13 (Lupishko et al. 1995). This asteroid was observed in detail from the ground in both optical and radar wavelength and from space by ChangE-2 spacecraft (Zheng et al. 2016). From space the boulders, grooves, and craters observed at the surface led to the conclusion that this asteroid is a rubble-pile asteroid (Zhu et al. 2014). Its irregular shape, long rotation period, and the complex rotation (not simple principal-axis rotation) imply a tumbling asteroid (Harris 1994).

(4179)Toutatis rotates with a precesion period of 7.38 days and a spin of 5.38 days (Mueller et al. 2002). These values are derived from photometric observations and are in agreement to the one derived from radar measurements (Ostro et al. 1999).

Considering that (4179)Toutatis has a rubble-pile structure and its rotation is more complex that a simple principal-axis, this asteroid makes a good candidate for parent body.

Six unpublished spectra of (4179)Toutatis (see Table A.1) are found in the MINUS database. We compute the taxonomy and we found the Sq, Q, or Sr taxonomic types for these spectra.

Neither the observation of ChangE-2, nor ground based observations allow us to observe dust meteoroids or cometary activity around this object.

The orbital evolution shows an unstable orbit $\left(T_{\mathrm{L}}=59 \mathrm{yr}\right.$, see Fig. D.2o).

Taurid Complex (TC). The Taurid Complex was intensively investigated (Steel et al. 1991; Babadzhanov et al. 2008; Popescu et al. 2014; Tubiana et al. 2015). TC is a complex of several showers. The most important are Northern Taurids (NTA) and Southern Taurids (STA). NTA has a $V_{\mathrm{g}}=28 \mathrm{~km} \mathrm{~s}^{-1}$ and a $\mathrm{ZHR}=5$ in 12 November, while STA has a $V_{\mathrm{g}}=$ $26.6 \mathrm{~km} \mathrm{~s}^{-1}$ and a ZHR $=5$ in 10 October. Several asteroids were suggested as parent body of this complex, together with the comet $\mathrm{P} /$ Enke.

For this complex we found four asteroids with physical data.

The asteroid 2004TG10 is associated with two meteor showers NTA and BTA respectively (see Table 1). This asteroid has a $V_{\mathrm{g}}=30.1 \mathrm{~km} \mathrm{~s}^{-1}$ slightly larger than the one of TC. Its albedo is around $0.02 \pm 0.04$ (Nugent et al. 2015).

The object 2010TU149 is associated with three meteor showers (see Table 1) and its $V_{\mathrm{g}}=27.7 \mathrm{~km} \mathrm{~s}^{-1}$. Its albedo is around $0.025 \pm 0.015$ (Mainzer et al. 2016).

The albedo of these two NEAs is an indicator of very dark surface commonly D, C, B, F-type objects.

2003UV11 was associated with three meteor showers (see Table 1). Its taxonomic class was determined as Q-type (DeMeo et al. 2014) while its albedo is $0.376 \pm 0.075$ (Mainzer et al. 2016).

2007RU17 was associated with medium probability to Southern Taurids (STA). A Q-type taxonomic class determined based on spectroscopic data (DeMeo et al. 2014).

We found three unpublished spectra from MINUS database, two for asteroid 2003UV11 (au2003UV11.sp94 and au2003UV11.sp95) and one for 2007RU17 (see Table A.1). By using M4AST curve matching methods for au2003UV11.sp94 
spectrum we found as relevant result the Sr-type (see Fig. C.1g). This type fits well the $2 \mu \mathrm{m}$ bands and the maximum at $1.5 \mu \mathrm{m}$. However, the band at $1 \mu \mathrm{m}$ it is matched by the Q-type. With the same settings 2007RU17 it is matched by the Q-type (see Fig. C.1j).

From the dynamical evolution of these asteroids, we classified as unstable orbits (2003UV11 $T_{\mathrm{L}}=86 \mathrm{yr}, 2004 \mathrm{TG} 11 T_{\mathrm{L}}=$ $75 \mathrm{yr}, 2007 \mathrm{RU} 17 T_{\mathrm{L}}=50 \mathrm{yr}$ and $2010 \mathrm{TU} 149 T_{\mathrm{L}}=59 \mathrm{yr}$, see Figs. D.11, m, D.2d, k.

$\tau$ Herculids (TAH). This meteor shower has a $V_{\mathrm{g}}=15 \mathrm{~km} \mathrm{~s}^{-1}$. We found four asteroids in our sample for which there are physical parameters available.

(3671)Dionysus was associated to TAH with medium probability. It is classified as $\mathrm{Cb}$ or $\mathrm{X}$ (Bus \& Binzel 2002; Binzel et al. 2004; Thomas et al. 2014) and its albedo is $0.18 \pm 0.09$ (Harris \& Lagerros 2002). We investigated the unpublished spectrum from the MINUS database. The spectrum of this asteroid has a poor SNR (signal to noise ratio). The feature around $1 \mu \mathrm{m}$ seems to be a data reduction artifact. The C-complex types match this spectrum (e.g., Ch, Fig. C.11).

In 1997 (3671)Dionysus passed between 17 million kilometers from the Earth. This close approach was observed and the photometry showed four events (May 1997 and 2-9 June 1997) which confirmed that Dionysus is a binary object (Mottola et al. 1997). The synodical periods of primary and the system allow us to infer that this object could be a complex rubble-pile structure.

2006HQ30 is a Sq or Q object (DeMeo et al. 2014). Its taxonomic class indicates that this object is more akin to ordinary chondrites composition. For this asteroid we found one unpublished spectra in MINUS database. From M4AST curve matching methods with band at $1 \mu \mathrm{m}$ it is matched by the Q-type (Fig. C.1k).

2014OY1 has an estimated albedo of $0.11 \pm 0.09$ (Nugent et al. 2015). Finally, the albedo of 2010GH65 is $0.241 \pm 0.055$ (Mainzer et al. 2011).

From the numerical integration we conclude that (3671)Dionysus and 2014OY1 have unstable orbits $\left(T_{\mathrm{L}}=\right.$ 52 respectively $72 \mathrm{yr}$, see Figs. D.2n, m), while for 2006HQ30 and $2010 \mathrm{GH} 65$ orbits are stable $\left(T_{\mathrm{L}}=443\right.$ respectively $2299 \mathrm{yr}$, see Figs. D.2a, i).

Daytime $\xi$ Sagittariids (XSA). This shower has a $V_{\mathrm{g}}=$ $24.4 \mathrm{~km} \mathrm{~s}^{-1}$. (325102)2008EY5 is the only object of our sample with an albedo of $0.124 \pm 0.027$ (Nugent et al. 2012). Its orbit is unstable $\left(T_{\mathrm{L}}=84 \mathrm{yr}\right.$, see Fig. D.2e).

Daytime $\zeta$ Perseids $(Z P E)$. This meteor shower has a $V_{\mathrm{g}}=$ $26.4 \mathrm{~km} \mathrm{~s}^{-1}$. The associated asteroid 1999RK45 has a Q-type taxonomic class (Tubiana et al. 2015) and its albedo is around 0.19 (Trilling et al. 2010). From orbital evolution we obtained a stable orbit $\left(T_{L}=465\right.$ yr, see Fig. D.1d).

$\alpha$ Virginids (AVB). This meteor shower has a $V_{\mathrm{g}}=$ $18.8 \mathrm{~km} \mathrm{~s}^{-1}$. We found two asteroids with physical data. (446791)1998SJ70 is a Q-type asteroid (DeMeo et al. 2014). The asteroid 2010FL has an albedo of $0.271 \pm 0.048$ (Mainzer et al. 2016).

We found one unpublished spectrum in MINUS database for (446791)1998SJ70. By using M4AST curve matching methods we found as relevant result the S-type. However, the best matched is Q-type with band at $1 \mu \mathrm{m}$ (see Fig. C.1m).

The orbit of this asteroid was classified as unstable $\left(T_{\mathrm{L}}=\right.$ 44 yr, see Fig. D.1c).

Corvids (COR). This meteor shower has a $V_{\mathrm{g}}=$ $8.7 \mathrm{~km} \mathrm{~s}^{-1}$. We found one asteroid with physical data, namely (162058)1997AE12. Its taxonomic class is Q-type
(DeMeo et al. 2014) and its albedo is $0.19 \pm 0.02$ (Nugent et al. 2015). Its orbit was classified as stable $\left(T_{\mathrm{L}}=172 \mathrm{yr}\right.$, see Fig. D.1b).

\subsection{Results of other similar studies}

Rudawska et al. (2012a) found five major meteoroid streams associated to eight asteroids. Asteroid 2005UW6 was associated with Taurid Complex. In our paper we find that this object is more akin to the Northern Taurids by only one metric. We obtained negative results for the association of 1997US2 and 2001XX103 claimed by Rudawska et al. (2012a).

Micheli (2013) associated asteroid 2007RU17 to Taurid Complex and observed the object for detecting its coma. The observations gave negative results. However he pointed out that the asteroid is part of Taurid Complex. In our work this asteroid was associated with medium probability to Southern Taurids.

Another clustering study performed by Šegon et al. (2014) associated 43 asteroids with inclination over $15^{\circ}$ with meteor showers using $D$-criteria metrics. In our calculations, we found only two common asteroids, namely (3200)Phaethon and 2009ST103. Another nine associations were found in our database but there are classified with low probability.

Żoasdek et al. (2016) studied the enhanced activity of the Southern Taurids detected on 31 October 2015 using $D$-criteria metrics and found three asteroids associated with fireballs, namely 2015TX24, 2005UR, and 2015TF50. In our analysis the asteroid 2015TX24 was associated with medium probability to both Southern Taurids and Daytime $\beta$ Taurids while 2005UR was identified as potential parent body by only one metric. Asteroid 2015 TF50 was not found between our candidates.

\subsection{Association of asteroids and meteorites}

Another interesting study is the association of asteroids and fall meteorites $^{12}$.

Popescu et al. (2014) studied the spectral properties of asteroids associated with TC. They found that five of their objects ((2201)Oljato, (4183)Cuno, (4486)Mithra, (5143)Heracles, and (6063)Jason) are S-type, with a spectrum similar with ordinary chondrites of petrologic type 6 (evolved surface). The spectrum of Farmington meteorite associated with TC, is similar with same of these asteroids spectra. Only (269690)1996RG3 is a C-type object which could be associated a primitive-cometary origin.

Birlan et al. (2015) associated HED meteorites with V-type PHA asteroids. Their result consist in two V-type PHA asteroids, (1981)Midas and 1997GL3, that can be associated with HED meteorites.

We made a search for fall meteorites. The search was made in the Meteoritical Bulletin Database ${ }^{13}$ over a period of $100 \mathrm{yr}$. In the searched interval we found 342 meteorites from which 62 of them occurs during the maximum activity of our 20 meteor showers associated with asteroids (maximum activity was obtained from IMO, Shower Calendar 2017 and the web site Meteor Shower Online ${ }^{14}$ based on Kronk (1988). Figure 5 shows the five categories of fall meteorites (Weisberg et al. 2006). We

\footnotetext{
${ }^{12}$ Fall meteorites - are those which were seen to fall form the sky and which were pursued and located successfully. This meteorites could be distinguished to the one found on the ground and related to any sighting. ${ }^{13}$ http://www. lpi.usra.edu/meteor/

${ }^{14}$ http://www . meteorshowersonline.com
} 

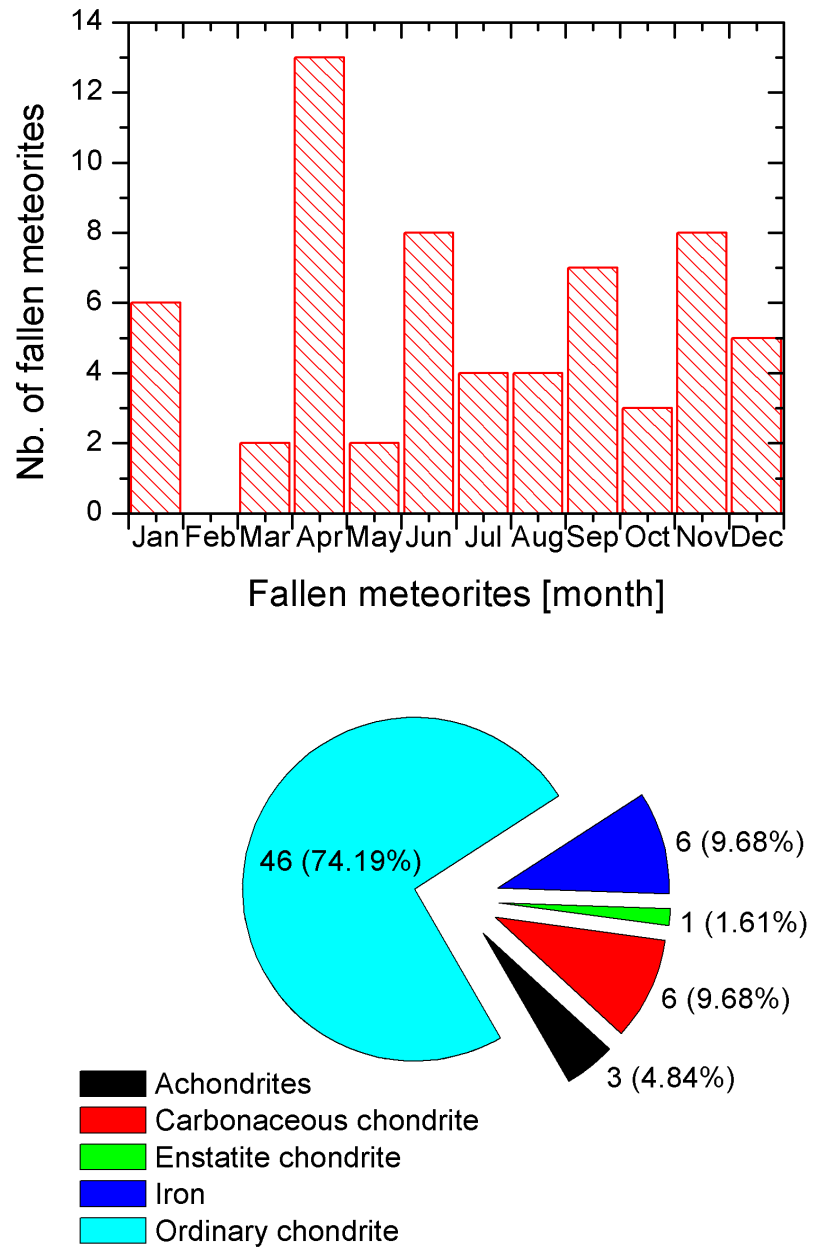

Fig. 5. Number of fall asteroids per month (top). Type of fall meteorites (bottom). Plotted are the 62 fall meteorites landing on Earth over a period of $100 \mathrm{yr}$ associated with maximum activity of meteor showers from our sample.

only used the maximum activity period, because the probability of a meteor falling from a particular stream is highest then.

From the total number of fall meteors associated $74.19 \%$ are ordinary chondrite meteorites (associated with all meteor showers), 9.68\% are iron meteorites (associated with APS, AVB, NCC, NTA, and SCC), 9.68\% are carbonaceous chondrite meteorites (associated with APS, COR, HVI, PPU, DSX, and NCC), 4.84\% achondrites meteorites (associated with AND, GEM, and BTA), and $1.61 \%$ enstatite meteorites (associated with AVB).

In a tentative approach we associated the fall meteorites with our associated asteroids that have physical data. The association between asteroid taxonomic class and meteoritic types is taken from Burbine (2016). This association is just a link from the fallen date of meteorites and maximum activity of the meteor shower, and it takes into consideration the fallen location of meteorites and radiants of meteor showers.

We note that hundreds of thousands of meteors are recorded annually but only about $30 \%$ of them are associated to meteor showers (Jenniskens et al. 2016b). Thus, we can only speculate on the origin of meteorites to meteor showers and their possible asteroid parent bodies.

In total we obtained eight asteroids that can be associated with 26 fall meteorites (see Table 2).

In January we found six fall meteorites (two carbonaceous chondrites, two irons and two ordinary chondrites) and one active meteor shower (NCC). From all of these, we can link two ordinary chondrites to asteroid (4179)Toutatis that is associated to NCC (maximum activity in 17 January). However from these meteorites, one is H-type and the other is L-type.

In April we have 13 fall meteorites (one carbonaceous chondrite, one estatite chondrite, two irons, and nine ordinary chondrites) and four active meteor showers (APS, AVB, PPU, HVI). From these fall meteorites we can link six from nine ordinary chondrites to asteroid 1998SJ70 that is associated to AVB (maximum activity in 7-18 April). Here we found three meteorites with H-type and three with L-type.

In June eight fall meteorites were recorded (one achondrite, one carbonaceous chondrite and six ordinary chondrites) and four active meteor showers were identified in our study (BTA, COR, TAH, ZPE). From these fall meteorites we can link four from six ordinary chondrites to two asteroids. 1999RK45 associated with ZPE (maximum activity on 9 June) and 2006HQ30 associated with TAH (maximum activity 8-11 June). For these asteroids we found one meteorite with H-type and three meteorites with L-type.

In July we found four fall meteorites (ordinary chondrites) and one active meteor shower (CAP). All the meteorites that fell in this month and one from August can be associated with asteroid 2001EC. This asteroid is associated to CAP (maximum activity in 30 July). However from these meteorites two have H-type and three have L-type.

In October we have three fall meteorites (ordinary chondrites) and one active meteor shower (STA). All the meteorites that fallen in this month can be associated with two asteroids. 2007RU17 and 2003UV11 are associated with STA (9 October). In this period we found only H-type meteorites. Here we can say that these asteroids can produce H-type meteorites.

In November we have eight fall meteorites (one achondrite, one iron, six ordinary chondrites) and two active meteor showers (AND and NTA). From these fall meteorites we can link all ordinary chondrites to two asteroids. We can link three fall meteorites to asteroid 2003FC5 that is associated with AND (maximum activity in 14-15 November) and three others to asteroid 2003UV11 that is associated with NTA (maximum activity in 12 November). For 2003FC5 we found meteorites with H-type(one) and L-type(two), and for 2003UV11 we found meteorites with H-type(one) and L-type(two).

However, the asteroid 2003UV11 is associated also with STA (October) where associated meteorites are H-type.

From our result (eight asteroids that may be associated with 26 fall meteorites), we searched for their physical data of asteroids and meteorites to see if our associations may be trusted. The main objective is to link physical data and see the association between the asteroid and the identified meteorites from Table 2. For this we made a comparison between spectra of asteroids and meteorites from Relab database using M4AST tools.

The asteroids (4179)Toutatis, 2006HQ30 and 2001EC have spectra in SMASS-MIT UH-IRTF, but we do not find spectra for associated meteorites (14 fall meteorites). For asteroid 1999RK45 we use a digitized spectrum from Tubiana et al. (2015) and we did not find any reliable match with the associated meteorites (four fall meteorites).

From all the associations we found three asteroids that may be associated to three meteorites (see Fig. B.1).

For Dwaleni meteorite we found three spectra that are similar with two spectra of asteroids. $c 1 t b 73$ spectrum was obtained in specific conditions: resolution: 10 , source angle: $30^{\circ}$, detect angle: $0^{\circ}$, temperature: room, atmosphere: ambient, amongst others. The object is of rock type with a maximum particle size 
B. A. Dumitru et al:: Association between meteor showers and asteroids using multivariate criteria

Table 2. 26 Fall meteorites that can be associated with 8 asteroids.

\begin{tabular}{|c|c|c|c|c|c|}
\hline Asteroid & Taxonomy & Fall meteor & Class & Type & Meteor ref. \\
\hline \multirow[t]{2}{*}{ (4179)Toutatis } & \multirow[t]{2}{*}{$\mathrm{S} / \mathrm{Sk} / \mathrm{Sq}$} & Seoni & Ordinary chondrite & H6 & 1 \\
\hline & & Lorton & Ordinary chondrite & L6 & 2 \\
\hline \multirow{6}{*}{ 1998SJ70 } & \multirow[t]{6}{*}{ Q } & Pétèlkolé & Ordinary chondrite & H5 & 3 \\
\hline & & Mason Gully & Ordinary chondrite & H5 & 2 \\
\hline & & Suizhou & Ordinary chondrite & L6 & 4 \\
\hline & & Xinglongquan & Ordinary chondrite & L3 & 5 \\
\hline & & Schenectady & Ordinary chondrite & $\mathrm{H} 4$ & 6 \\
\hline & & Mifflin & Ordinary chondrite & L5 & 2 \\
\hline \multirow[t]{4}{*}{ 1999RK45 and 2006HQ30 } & \multirow[t]{4}{*}{$\mathrm{Q}$ and $\mathrm{Q} / \mathrm{Sq}$} & Ste. Marguerite & Ordinary chondrite & $\mathrm{H} 4$ & 7 \\
\hline & & Hökmark & Ordinary chondrite & L4 & 8 \\
\hline & & Lanxi & Ordinary chondrite & L6 & 9 \\
\hline & & Sheyang & Ordinary chondrite & L6 & 4 \\
\hline \multirow[t]{5}{*}{ 2001EC } & \multirow[t]{5}{*}{$\mathrm{Sq}$} & Maigatari-Danduma & Ordinary chondrite & $\mathrm{H} 5 / 6$ & 10 \\
\hline & & Jodiya & Ordinary chondrite & L5 & 11 \\
\hline & & Sidi Ali Ou Azza & Ordinary chondrite & L4 & 12 \\
\hline & & Kokubunji & Ordinary chondrite & L6 & 4 \\
\hline & & Wuan & Ordinary chondrite & H6 & 13 \\
\hline \multirow[t]{3}{*}{ 2007RU17 and 2003UV11 } & \multirow[t]{3}{*}{$\mathrm{Q}$ and $\mathrm{Q}$} & Ishinga & Ordinary chondrite & $\mathrm{H}$ & 14 \\
\hline & & Peekskill & Ordinary chondrite & H6 & 15 \\
\hline & & Dwaleni & Ordinary chondrite & H4-6 & 16 \\
\hline \multirow[t]{3}{*}{2003 FC5 } & \multirow[t]{3}{*}{$\mathrm{S}$} & Kingai & Ordinary chondrite & H6 & 17 \\
\hline & & New Halfa & Ordinary chondrite & L4 & 18 \\
\hline & & Wethersfield (1982) & Ordinary chondrite & L6 & 19 \\
\hline \multirow[t]{3}{*}{ 2003UV11 } & \multirow[t]{3}{*}{ Q } & Kamargaon & Ordinary chondrite & L6 & 20 \\
\hline & & Salzwedel & Ordinary chondrite & LL5 & 21 \\
\hline & & Chiang Khan & Ordinary chondrite & H6 & 22 \\
\hline
\end{tabular}

Notes. This association is just a link from the fallen date of meteorites and maximum activity of the meteor shower. The asteroids associated to the meteor shower are only the asteroids with physical data, considering the fallen location of meteorite and radiant of meteor shower.

References. (1) Clarke (1974); (2) Garvie (2012); (3) Russell et al. (2003); (4) Graham (1987); (5) http://www.lpi.usra.edu/meteor/ metbull . php?code=62494; (6) Fleischer et al. (1970); (7) Telus et al. (2016); (8) Krinov (1961); (9) Graham (1989); (10) Russell et al. (2005); (11) Weisberg et al. (2008); (12) Chennaoui Aoudjehane et al. (2016); (13) Graham (1988); (14) Krinov (1958); (15) Wlotzka (1993); (16) Clarke (1971); (17) Krinov (1970); (18) Wlotzka (1995); (19) Graham (1984); (20) http: //www . lpi . usra . edu/meteor/metbull . php?code=63102; (21) Graham (1986); (22) Graham (1982).

of $150 \mu \mathrm{m}$. This spectrum was associated with asteroids spectra $a u 2007 R U 17 . s p 94$ (see Fig. B.1a) and au2003UV11.sp95 (see Fig. B.1c). clmb03 spectrum was obtained in the same condition as $c 1 t b 73$. But this rock type object has the particle size between 25 and $250 \mu \mathrm{m}$. This spectrum was associated with asteroid spectra au2003UV11.sp 94 (see Fig. B.1b), and is the best association from this meteorite.

For the Chiang Khan meteorite we found similarities with one asteroid, namely 2003UV11. Its spectrum was obtained in the same conditions as $c 1 t b 73$ and the object has a rock type with its maximum particle size is $150 \mu \mathrm{m}$. This spectrum was associated with two spectra of asteroid 2003UV11, au2003UV11.sp94 in Fig. B.1e and $a u 2003 U V 11 . s p 95$ in Fig. B.1f. The best association at this meteorite is with spectra au2003UV11.sp 94 .

For the Schenectady meteorite we found similarities with one asteroid, namely 1998SJ70. This spectrum was obtained in the same conditions, preview spectra, and its rock type object has a maximum particle size of $150 \mu \mathrm{m}$. The spectrum is similar with the asteroid spectra $a u 1998 S$ J70.sp74 (see Fig. B.1d).

For the Wethersfield (1982) meteorite we found similarities with one asteroid, namely 2003FC5. For this meteorite we found one spectrum, namely $c 1 t b 147$. The sample is of rocky type with a maximum particle size of $150 \mu \mathrm{m}$. The match of 2003FC5 and the Wethersfield meteorite is presented in Fig. B1g. We observe a discrepancy between asteroids and meteorite spectra in particular around $1 \mu \mathrm{m}$ abortion band, which can be explained if we consider space-weathering mechanisms (Brunetto et al. 2006; Pieters et al. 2000).

\section{Conclusions}

Three metrics $\left(D_{\mathrm{ACS}}, D_{\mathrm{SH}}\right.$ and $\left.D_{\mathrm{H}}\right)$ were used for finding asteroids that can be associated with meteor showers. The results of clustering were corroborated with dynamical backward integration of the selected objects in order to analyze their stability.

206 near-Earth asteroids were found to be associated with 28 meteor showers by at least two metrics. About $26 \%$ of the associated asteroids are classified as PHA objects.

In our results we found asteroids that are already associated with meteor showers, such as (3200)Phaethon with Geminids (GEM), 2005UD with Daytime Sextantids (DSX), 2004TG10 was associated with Northern Taurids (NTA).

Of our 206 asteroids, only 31 have sparse physical parameters. These objects were analyzed in terms of their potential to produce meteoroids. Thus, the fast-rotator asteroid 2007LW19 is most probably of monolithic structure and is unlikely to produce meteoroids. At the opposite end of the scale, slow-rotators and tumbling asteroids are more akin to be rubble-pile objects, thus weak gravitational instabilities or non-gravitational forces could easily detach meteoroids from these bodies. We notice here the binary asteroid 2000UG11 associated with AND, and 
the tumbling asteroid (4179)Toutatis (rubble-pile body) associated with OCC.

Analysis of available spectra for some of the samples reveal similarities on the mineralogy of the parent body and the possible associated fall meteorites.

We searched fall meteorites that can be associated with our sample of meteor showers and we found 62 meteorites. From the total possible associated meteorites, $74.19 \%$ are ordinary chondrites, $4.84 \%$ are achondrites, $9.6 \%$ are carbonaceous chondrites, $9.68 \%$ are iron and $1.61 \%$ are enstatite chondrites.

Acknowledgements. We would like to thank Dr Galina Ryabova and the anonymous referee for the very useful and constructive reports which helped improve the paper. The work of B.A. Dumitru, M. Popescu, D.A. Nedelcu was financed by a grant of the Romanian National Authority for Scientific Research and Innovation, CNCS - UEFISCDI, project number PN-II-RU-TE-2014-4-2199. Also this work was supported by Paris Observatory PICS-PASSO program, Institute of Space Science and Astronomical Institute of the Romanian Academy. All (o part) of the data utilized in this publication were obtained and made available by the MIT-UH-IRTF Joint Campaign for NEO Reconnaissance. The IRTF is operated by the University of Hawaii under Cooperative Agreement No. NCC 5-538 with the National Aeronautics and Space Administration, Office of Space Science, Planetary Astronomy Program. The MIT component of this work is supported by NASA grant 09-NEOO009-0001, and by the National Science Foundation under Grants Nos. 0506716 and 0907766. Any opinions, findings, and conclusions or recommendations expressed in this material are those of the author(s) and do not necessarily reflect the views of NASA or the National Science Foundation.

\section{References}

Abedin, A., Wiegert, P., Pokorný, P., \& Brown, P. 2017, Icarus, 281, 417 Alvarez, L. W., Alvarez, W., Asaro, F., \& Michel, H. V. 1980, Science, 208, 1095 Asher, D. J., Clube, S. V. M., \& Steel, D. I. 1993, MNRAS, 264, 93 Babadzhanov, P. B., Williams, I. P., \& Kokhirova, G. I. 2008, MNRAS, 386, 1436

Binzel, R. P., Lupishko, D., di Martino, M., Whiteley, R. J., \& Hahn, G. J. 2002, in Physical Properties of Near-Earth Objects, eds. W. F. Bottke, Jr., A. Cellino, P. Paolicchi, \& R. P. Binzel, 255

Binzel, R. P., Rivkin, A. S., Stuart, J. S., et al. 2004, Icarus, 170, 259

Binzel, R. P., Morbidelli, A., Merouane, S., et al. 2010, Nature, 463, 331

Birlan, M., Popescu, M., Nedelcu, D. A., et al. 2015, A\&A, 581, A3

Birlan, M., Popescu, M., Irimiea, L., \& Binzel, R. 2016, in AAS/Division for Planetary Sciences Meeting Abstracts, 48, 325.17

Brunetto, R., Vernazza, P., Marchi, S., et al. 2006, Icarus, 184, 327

Burbine, T. H. 2016, in Lun. Planet. Sci. Conf., 47, 2425

Bus, S. J., \& Binzel, R. P. 2002, Icarus, 158, 106

Chennaoui Aoudjehane, H., Agee, C. B., Aaranson, A., \& Bouragaa, A. 2016, LPI Contributions, 1921, 6120

Chesley, S. R., \& Milani, A. 1999, in AAS/Division for Planetary Sciences Meeting Abstracts, 31, 28.06

Clarke, Jr., R. S. 1971, Meteoritics, 6, 111

Clarke, Jr., R. S. 1974, Meteoritics, 9, 101

Clube, S. V. M., \& Napier, W. M. 1984, MNRAS, 211, 953

Davies, J. K., Harris, A. W., Rivkin, A. S., et al. 2007, Icarus, 186, 111

DeMeo, F. E., Binzel, R. P., Slivan, S. M., \& Bus, S. J. 2009, Icarus, 202, 160

DeMeo, F. E., Binzel, R. P., \& Lockhart, M. 2014, Icarus, 227, 112

Drummond, J. D. 1981, Icarus, 45, 545

Farinella, P., Foschini, L., Froeschlé, C., et al. 2001, A\&A, 377, 1081

Fleischer, R. L., Lifshin, E., Price, P. B., et al. 1970, Icarus, 12, 402

Fornasier, S., Belskaya, I. N., Shkuratov, Y. G., et al. 2006, A\&A, 455, 371

Fulchignoni, M., Birlan, M., \& Antonietta Barucci, M. 2000, Icarus, 146, 204

Garvie, L. A. J. 2012, Meteor. Planet. Sci., 47, 1887

Graham, A. L. 1982, Meteoritics, 17, 93

Graham, A. L. 1984, Meteoritics, 19, 49

Graham, A. L. 1986, Meteoritics, 21, 309

Graham, A. L. 1987, Meteoritics, 22, 157

Graham, A. L. 1988, Meteoritics, 23, 171

Graham, A. L. 1989, Meteoritics, 24, 57

Gustafson, B. A. S. 1989, A\&A, 225, 533

Harris, A. W. 1994, Icarus, 107, 209

Harris, A. W., \& Lagerros, J. S. V. 2002, in Asteroids in Thermal Infrared, eds. W. F. Bottke, Jr., A. Cellino, P. Paolicchi, \& R. P. Binzel, 205

Hildebrand, A. R., Penfield, G. T., Kring, D. A., et al. 1991, Geology, 19, 867
Jenniskens, P. 2004, AJ, 127, 3018

Jenniskens, P. 2006, Meteor Showers and their Parent Comets (Cambridge, UK: Cambridge University Press)

Jenniskens, P., Nénon, Q., Albers, J., et al. 2016a, Icarus, 266, 331

Jenniskens, P., Nénon, Q., Gural, P. S., et al. 2016b, Icarus, 266, 355

Jewitt, D., \& Hsieh, H. 2006, AJ, 132, 1624

Jewitt, D., \& Li, J. 2010, AJ, 140, 1519

Jewitt, D., Li, J., \& Agarwal, J. 2013, ApJ, 771, L36

Jewitt, D., Hsieh, H., \& Agarwal, J. 2015, in The Active Asteroids, eds. P. Michel, F. E. DeMeo, \& W. F. Bottke, 221

Jones, J., Poole, L. M. G., \& Webster, A. R. 2016, MNRAS, 455, 3424

Jopek, T. J. 1993, Icarus, 106, 603

Jopek, T. J., \& Jenniskens, P. M. 2011, in Meteoroids: The Smallest Solar System Bodies, eds. W. J. Cooke, D. E. Moser, B. F. Hardin, \& D. Janches, 7

Jopek, T. J., \& Kaňuchová, Z. 2014, Meteoroids, 2013, 353

Jopek, T. J., \& Williams, I. P. 2013, MNRAS, 430, 2377

Jopek, T. J., Rudawska, R., \& Bartczak, P. 2008, Earth Moon Planets, 102, 73

Jopek, T. J., Koten, P., \& Pecina, P. 2010, MNRAS, 404, 867

Kinoshita, D., Ohtsuka, K., Sekiguchi, T., et al. 2007, A\&A, 466, 1153

Krinov, E. L. 1958, Meteoritical Bulletin Archive, 8

Krinov, E. L. 1961, Meteoritical Bulletin Archive, 128

Krinov, E. L. 1970, Meteoritics, 5, 85

Kronk, G. W. 1988, Meteor showers, A descriptive catalog (Enslow Publ.)

Kulik, L. A. 1938, Leaflet of the Astronomical Society of the Pacific, 3, 78

Kwiatkowski, T., Buckley, D. A. H., O’Donoghue, D., et al. 2010, A\&A, 509, A94

Li, J., \& Jewitt, D. 2013, AJ, 145, 154

Licandro, J., Campins, H., Mothé-Diniz, T., Pinilla-Alonso, N., \& de León, J. 2007, A\&A, 461, 751

Lindblad, B. A. 1971a, Smithsonian Contributions to Astrophysics, 12, 14

Lindblad, B. A. 1971b, Smithsonian Contributions to Astrophysics, 12, 1

Lupishko, D. F., Vasilyev, S. V., Efimov, J. S., \& Shakhovskoj, N. M. 1995, Icarus, 113, 200

Mainzer, A., Grav, T., Bauer, J., et al. 2011, ApJ, 743, 156

Mainzer, A., Bauer, J., Grav, T., et al. 2014, ApJ, 784, 110

Mainzer, A. K., Bauer, J. M., Cutri, R. M., et al. 2016, NASA Planetary Data System, 247

Margot, J. L., Nolan, M. C., Benner, L. A. M., et al. 2002, Science, 296, 1445

Meng, H., Zhu, J., Gong, X., et al. 2004, Icarus, 169, 385

Micheli, M. 2013, Ph.D. Thesis, University of Hawai'i at Manoa

Micheli, M., Bernardi, F., \& Tholen, D. J. 2008, MNRAS, 390, L6

Miskotte, K. 2016, eMeteorNews, 1, 52

Mottola, S., Hahn, G., Pravec, P., \& Sarounova, L. 1997, IAU Circ., 6680

Mueller, B. E. A., Samarasinha, N. H., \& Belton, M. J. S. 2002, Icarus, 158, 305

Napier, W. M. 2010, MNRAS, 405, 1901

Nedelcu, D. A., Birlan, M., Souchay, J., et al. 2010, A\&A, 509, A27

Nedelcu, D. A., Birlan, M., Popescu, M., Bădescu, O., \& Pricopi, D. 2014, A\&A, 567, L7

Nesvorný, D., \& Vokrouhlický, D. 2006, AJ, 132, 1950

Nugent, C. R., Mainzer, A., Masiero, J., Grav, T., \& Bauer, J. 2012, AJ, 144, 75

Nugent, C. R., Mainzer, A., Masiero, J., et al. 2015, ApJ, 814, 117

Ohtsuka, K., Sekiguchi, T., Kinoshita, D., \& Watanabe, J. 2005, Central Bureau Electronic Telegrams, 283

Ohtsuka, K., Sekiguchi, T., Kinoshita, D., et al. 2006, A\&A, 450, L25

Ostro, S. J., Hudson, R. S., Rosema, K. D., et al. 1999, Icarus, 137, 122

Pierazzo, E., Hahmann, A. N., \& Sloan, L. C. 2003, Astrobiology, 3, 99

Pieters, C. M., Taylor, L. A., Noble, S. K., et al. 2000, Meteor. Planet. Sci., 35, 1101

Polishook, D., Moskovitz, N., Binzel, R. P., et al. 2016, Icarus, 267, 243

Popescu, M., Birlan, M., \& Nedelcu, D. A. 2012, A\&A, 544, A130

Popescu, M., Birlan, M., Nedelcu, D. A., Vaubaillon, J., \& Cristescu, C. P. 2014, A\&A, 572, A106

Popova, O. P., Jenniskens, P., Emel'yanenko, V., et al. 2013, Science, 342, 1069

Porubčan, V., Williams, I. P., \& Kornoš, L. 2004, Earth Moon Planets, 95, 697

Porubčan, V., Kornoš, L., \& Williams, I. P. 2006, Contributions of the Astronomical Observatory Skalnate Pleso, 36, 103

Pravec, P., Scheirich, P., Kušnirák, P., et al. 2006, Icarus, 181, 63

Reddy, V., Dunn, T. L., Thomas, C. A., Moskovitz, N. A., \& Burbine, T. H. 2015, in Mineralogy and Surface Composition of Asteroids, eds. P. Michel, F. E. DeMeo, \& W. F. Bottke, 43

Rendtel, J. 2004, Earth Moon Planets, 95, 27

Richardson, D. C., Michel, P., Walsh, K. J., \& Flynn, K. W. 2009, Planet. Space Sci., 57, 183

Rubin, A. E., \& Grossman, J. N. 2010, Meteor. Planet. Sci., 45, 114

Rudawska, R., Vaubaillon, J., \& Atreya, P. 2012a, A\&A, 541, A2

Rudawska, R., Vaubaillon, J., \& Jenniskens, P. 2012b, in Eur. Planet. Sci. Congress 2012, EPSC2012-886

Russell, S. S., Zipfel, J., Folco, L., et al. 2003, Meteor. Planet. Sci., 38, A189 
B. A. Dumitru et al.: Association between meteor showers and asteroids using multivariate criteria

Russell, S. S., Zolensky, M., Righter, K., et al. 2005, Meteor. Planet. Sci., 40, A201

Ryabova, G. O. 2007, MNRAS, 375, 1371

Ryabova, G. O. 2012, MNRAS, 423, 2254

Ryabova, G. O. 2016, MNRAS, 456, 78

Ryabova, G. O., Pleshanova, A. V., \& Konstantinov, V. S. 2008, Sol. Syst. Res. 42,335

Scheeres, D. J. 2007, Icarus, 189, 370

Schiaparelli, G. V. 1867, MNRAS, 27, 246

Šegon, D., Gural, P., Andreić, Ž., et al. 2014, Meteoroids 2013 (A. M. University Press)

Shapley, H. 1930, Flights from chaos; a survey of material systems from atoms to galaxies, adapted from lectures at the College of the city of New York, Class of 1872 foundation (Whittlesey house, McGraw-Hill book company, inc.)

Smit, J. 1980, Nature, 285, 198

Southworth, R. B., \& Hawkins, G. S. 1963, Smithsonian Contributions to Astrophysics, 7, 261

Steel, D. I., Asher, D. J., \& Clube, S. V. M. 1991, MNRAS, 251, 632

Tancredi, G. 1998, Celest. Mech. Dyn. Astron., 70, 181

Tancredi, G., Sánchez, A., \& Roig, F. 2001, AJ, 121, 1171

Telus, M., Alexander, C. M. O., Hauri, E. H., \& Wang, J. 2016, in Lun. Planet. Sci. Conf., 47, 1742

Thomas, C. A., Emery, J. P., Trilling, D. E., et al. 2014, Icarus, 228, 217

Toon, O. B., Zahnle, K., Morrison, D., Turco, R. P., \& Covey, C. 1997, Rev. Geophys., 35, 41

Trilling, D. E., Mueller, M., Hora, J. L., et al. 2010, AJ, 140, 770

Tubiana, C., Snodgrass, C., Michelsen, R., et al. 2015, A\&A, 584, A97

Valsecchi, G. B., Jopek, T. J., \& Froeschle, C. 1999, MNRAS, 304, 743
Vaubaillon, J., Colas, F., \& Jorda, L. 2005, A\&A, 439, 751

Walsh, K. J., Delbo', M., Mueller, M., Binzel, R. P., \& DeMeo, F. E. 2012, ApJ, 748, 104

Weisberg, M. K., McCoy, T. J., \& Krot, A. N. 2006, in Systematics and Evaluation of Meteorite Classification, eds. D. S. Lauretta, \& H. Y. McSween, 19

Weisberg, M. K., Smith, C., Benedix, G., et al. 2008, Meteor. Planet. Sci., 43, 1551

Weiss, E. 1868, Astron. Nachr., 72, 81

Weissman, P. R., A'Hearn, M. F., Rickman, H., \& McFadden, L. A. 1989, in Asteroids II, eds. R. P. Binzel, T. Gehrels, \& M. S. Matthews, 880

Whipple, F. L. 1983, IAU Circ., 3881

Whipple, F. L., \& El-Din Hamid, S. 1952, Helwan Institute of Astronomy and Geophysics Bulletins, 41, 3

Wiegert, P., Vaubaillon, J., \& Campbell-Brown, M. 2009, Icarus, 201, 295

Williams, I. P. 2011, Astron. Geophys., 52, 2.20

Williams, I. P., Ryabova, G. O., Baturin, A. P., \& Chernitsov, A. M. 2004, MNRAS, 355, 1171

Wisniewski, W. Z., Michałowski, T. M., Harris, A. W., \& McMillan, R. S. 1997, Icarus, 126, 395

Wlotzka, F. 1993, Meteoritics, 28, 692

Wlotzka, F. 1995, Meteoritics, 30, 792

Zellner, B., Tholen, D. J., \& Tedesco, E. F. 1985, Icarus, 61, 355

Zheng, C., Ping, J., \& Wang, M. 2016, Icarus, 278, 119

Zhu, M.-H., Fa, W., Ip, W.-H., et al. 2014, Geophys. Res. Lett., 41, 328

Żołądek, P., Olech, A., Wiśniewski, M., et al. 2016, in Proc. of the Int. Meteor Conf. Egmond, The Netherlands, 2-5 June 2016, eds. A. Roggemans, \& P. Roggemans, 358 


\section{Appendix A: Objects found in SMASS-MIT UH-IRTF and processed with M4AST}

Table A.1. Spectra found in SMASS-MIT UH-IRTF database and the taxonomic classes associated using M4AST.

\begin{tabular}{|c|c|c|c|c|c|}
\hline Object & File name & $\lambda_{\min }$ & $\lambda_{\max }$ & Taxonomy class & Fig. \\
\hline (4179)Toutatis & $a 004179 . s p 03$ & 0.435 & 2.475 & $\mathrm{Sr}$ & C.1a \\
\hline (4179)Toutatis & $a 004179 . s p 30$ & 0.435 & 2.475 & $\mathrm{Sq}$ & C. $1 \mathrm{~b}$ \\
\hline (4179)Toutatis & $a 004179 . s p 73$ & 0.435 & 2.475 & $\mathrm{Sq}$ & C. $1 \mathrm{c}$ \\
\hline (4179)Toutatis & $a 004179 . s p 74$ & 0.435 & 2.475 & Q & C. $1 \mathrm{~d}$ \\
\hline (4179)Toutatis & $a 004179 . s p 115 n 1$ & 0.8 & 2.475 & $\mathrm{Sq}$ & C.1e \\
\hline (4179)Toutatis & $a 004179 . s p 116$ & 0.8 & 2.475 & $\mathrm{Sq}$ & C.1f \\
\hline 2003UV11 & аu2003UV11.sp94 & 0.78 & 2.475 & $\mathrm{Sr}$ & C. $1 \mathrm{~g}$ \\
\hline 2003UV11 & au2003UV11.sp95 & 0.78 & 2.475 & Q & C. $1 \mathrm{~h}$ \\
\hline 2000DO1 & au2000DO1visir8.visir & 0.435 & 2.475 & $\mathrm{~V}$ & C.1i \\
\hline 2007RU17 & au2007RU17.sp94 & 0.78 & 2.475 & $\mathrm{Q}$ & C. $1 \mathrm{j}$ \\
\hline 2006HQ30 & au2006HQ30.sp51 & 0.78 & 2.2 & Q & C. $1 \mathrm{k}$ \\
\hline (3671)Dionysus & $a 003671 . s p 91$ & 0.435 & 2.45 & $\mathrm{Ch}$ & C.11 \\
\hline 1998SJ70 & au1998S J70.sp74 & 0.78 & 2.2 & $\mathrm{Q}$ & C. $1 \mathrm{~m}$ \\
\hline 2003FC5 & a267729.sp98 & 0.78 & 2.4 & $\mathrm{~K}$ & C. $1 \mathrm{n}$ \\
\hline
\end{tabular}

Appendix B: Asteroid spectra compared with meteorite spectra (Relab)

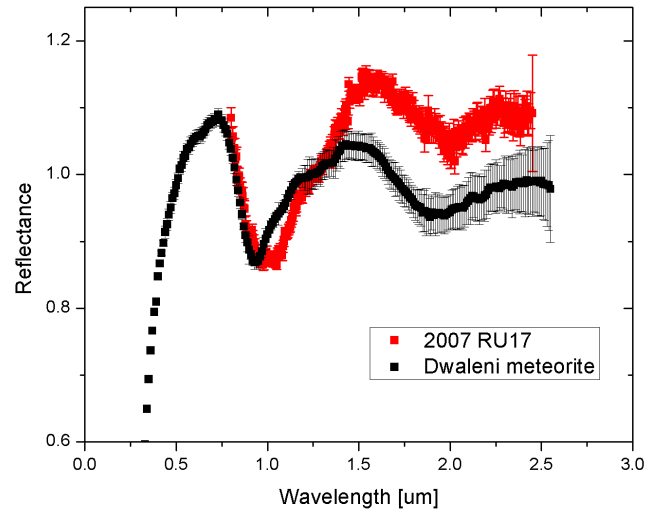

(a)

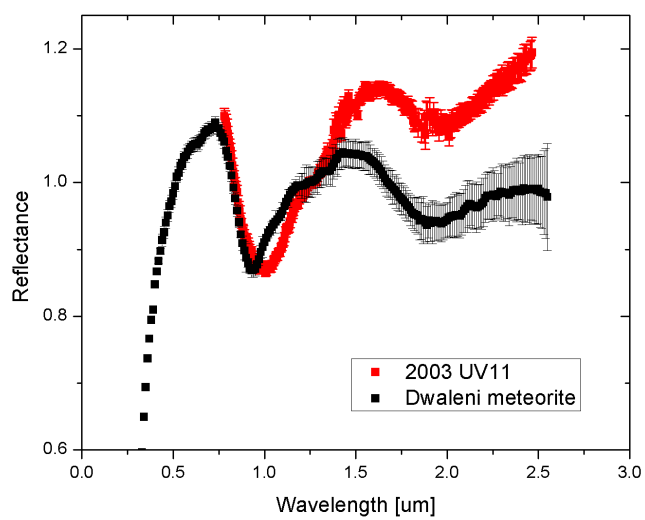

(c)

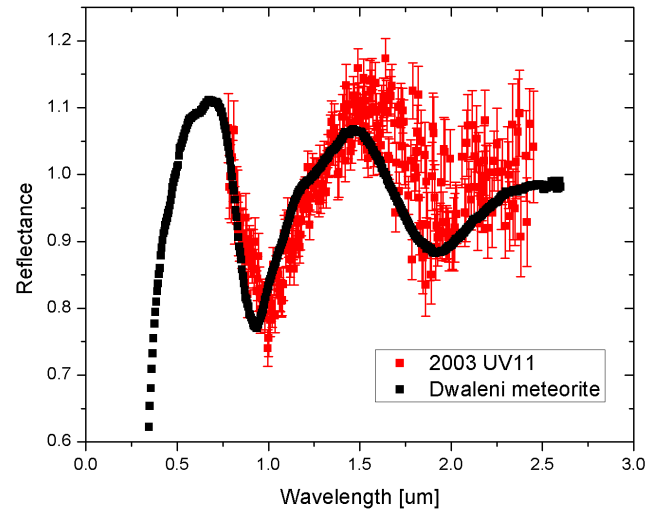

(b)

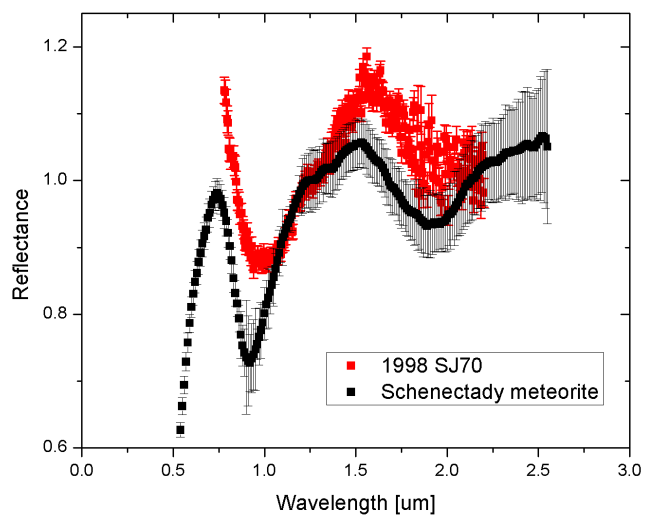

(d)

Fig. B.1. Spectra comparison between meteorites and asteroids associated to meteor showers. Both asteroid and meteorite spectra were normalized to $1.25 \mu \mathrm{m}$. Panel a: 2007RU17 (au2007RU17.sp94) compared with Dwaleni meteorite (c1tb73). Panel b: 2003UV11 (au2003UV11.sp94) compared with Dwaleni meteorite (clmb03). Panel c: 2003UV11 (au2003UV11.sp95) compared with Dwaleni meteorite (c1tb73). Panel d: 1998SJ70 (au1998S J70.sp74) compared with Schenectady meteorite (c1tb83). Panel e: 2003UV11 (au2003UV11.sp94) compared with Chiang Khan meteorite (c1tb132). Panel f: 2003UV11 (au2003UV11.sp95) compared with Chiang Khan meteorite (c1tb132). Panel g: 2003FC5 (a267729.sp98) compared with Wethersfield (1982) meteorite ( $c 1 T$ B147). 
B. A. Dumitru et al.: Association between meteor showers and asteroids using multivariate criteria

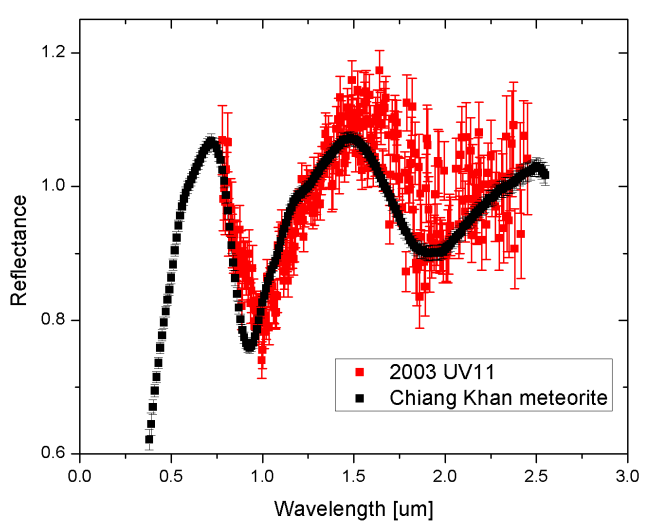

(e)

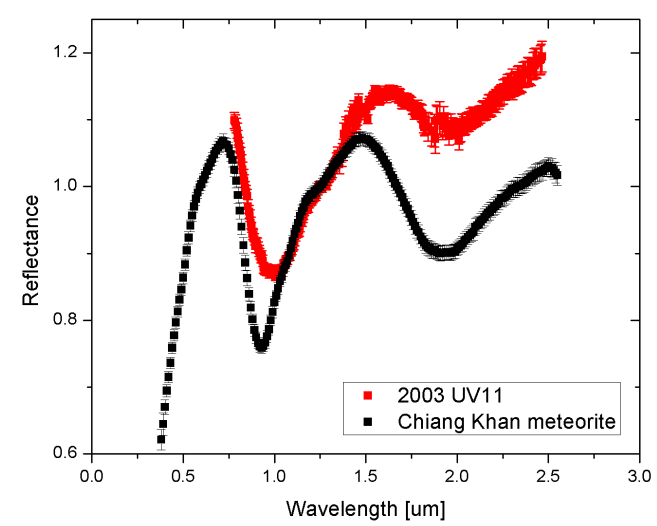

(f)

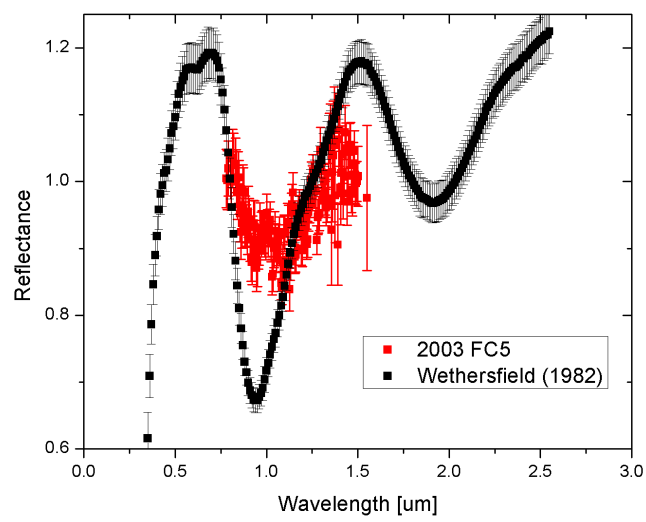

(g)

Fig. B.1. continued. 


\section{Appendix C: Spectra classification with Bus-DeMeo taxonomy using M4AST}

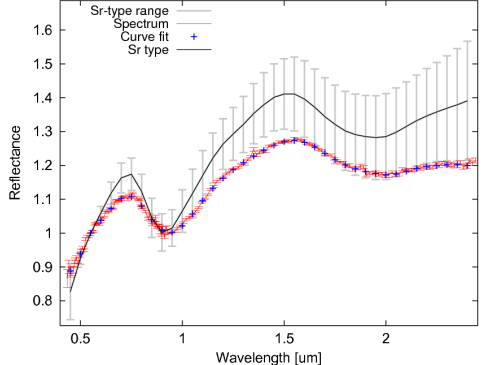

(a) 004179.sp03

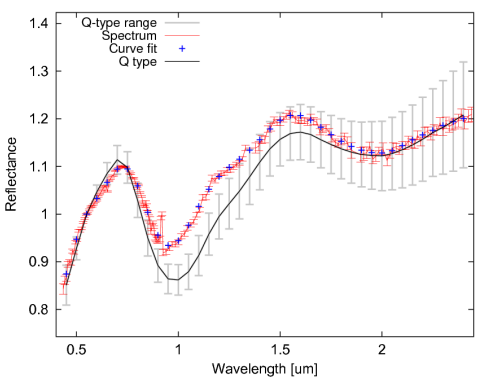

(d) $a 004179 . s p 74$

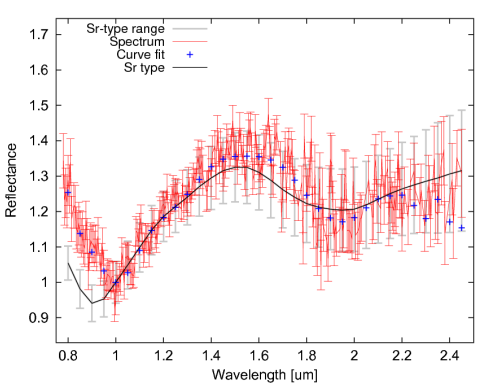

(g) au2003UV11.sp94 (1 $\mu \mathrm{m})$

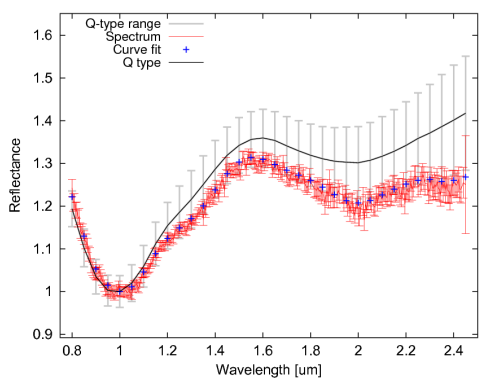

(j) au2007RU17.sp94 (1 $\mu \mathrm{m})$

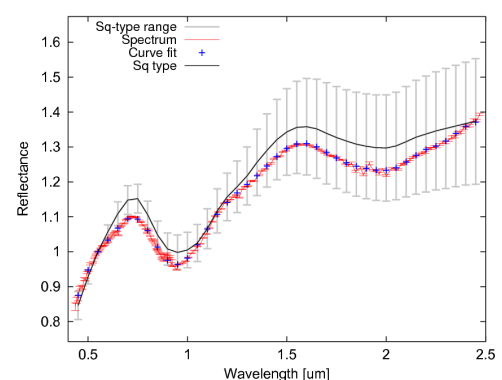

(b) $a 004179 . s p 30$

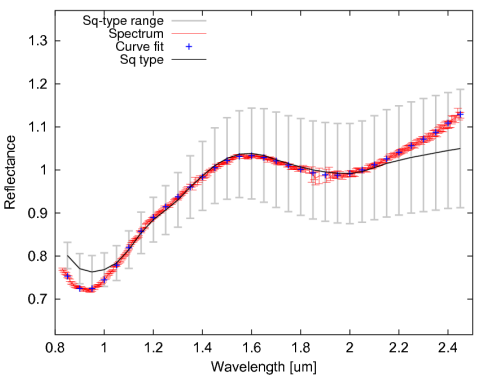

(e) $a 004179 . s p 115 n 1$

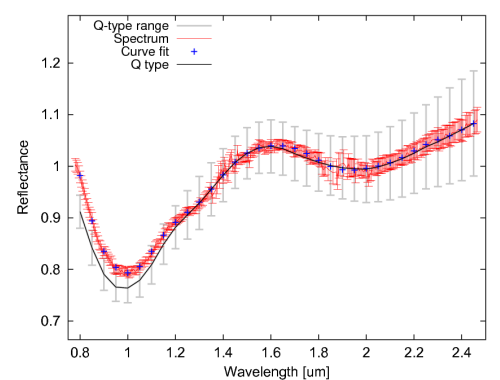

(h) au2003UV11.sp 95

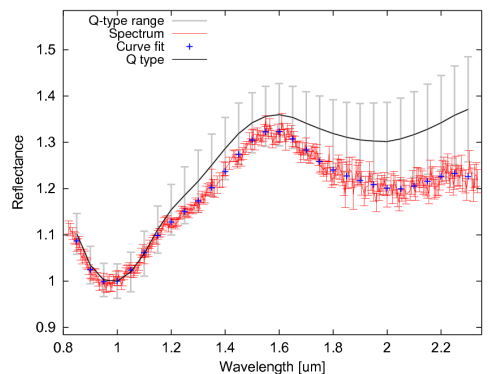

(k) au2006HQ30.sp51 (1 $\mu \mathrm{m})$

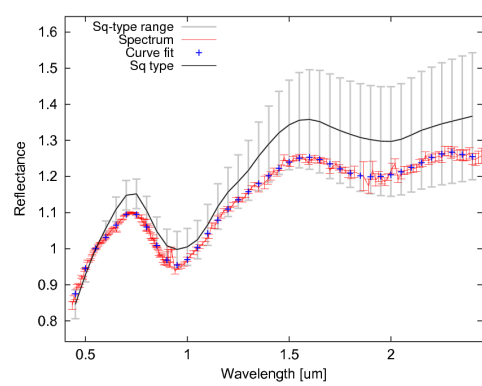

(c) $a 004179 . s p 73$

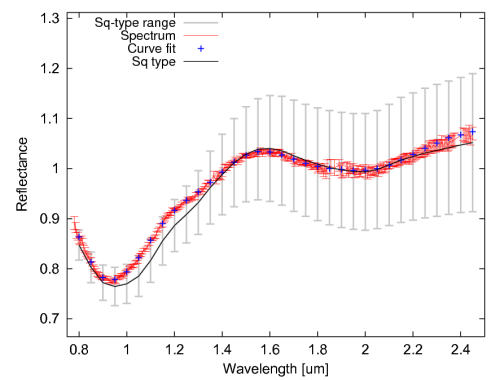

(f) $a 004179 . s p 116$

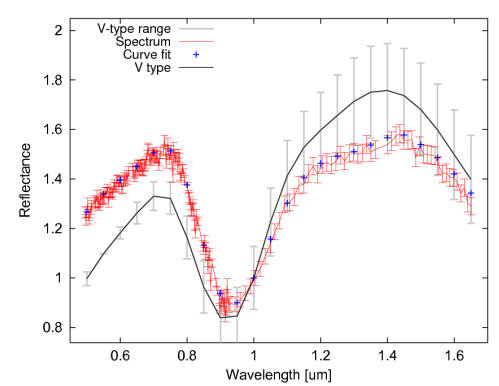

(i) au2000DO1visir8.visir $(1 \mu \mathrm{m})$

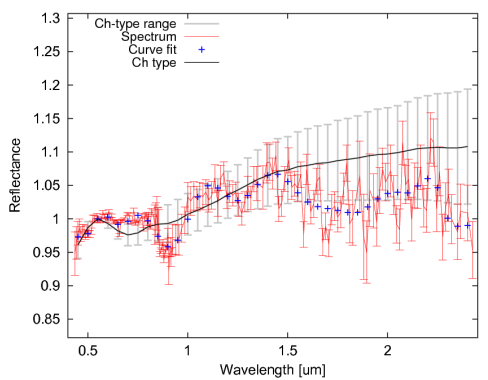

(1) $a 003671 . s p 91$

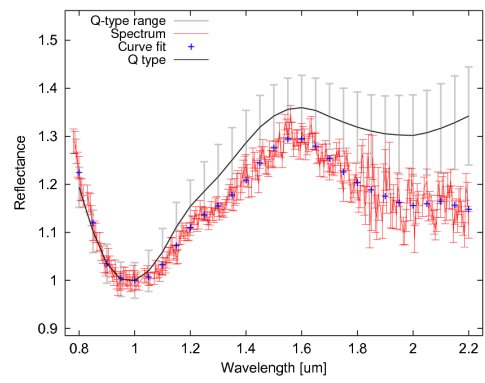

(m) au1998S J70.sp74 (1 $\mu \mathrm{m})$

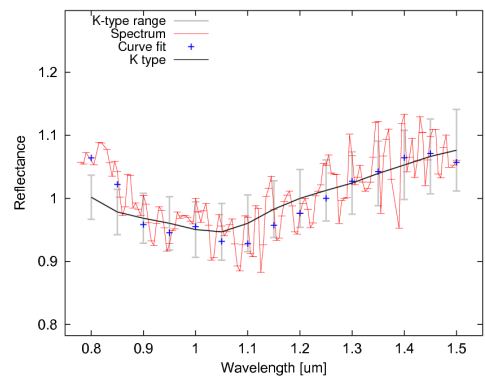

(n) $a 267729 . s p 98$

Fig. C.1. Taxonomic classification of selected asteroids. If the visible part was available the spectra were normalized to $0.55 \mu \mathrm{m}$, otherwise for the NIR part only we normalized at $1.25 \mu \mathrm{m}$ (if not specified). In few cases we normalized to $1 \mu \mathrm{m}$ to show the band similarities. 
B. A. Dumitru et al.: Association between meteor showers and asteroids using multivariate criteria

\section{Appendix D: Asteroids perihelion (q) evolution}

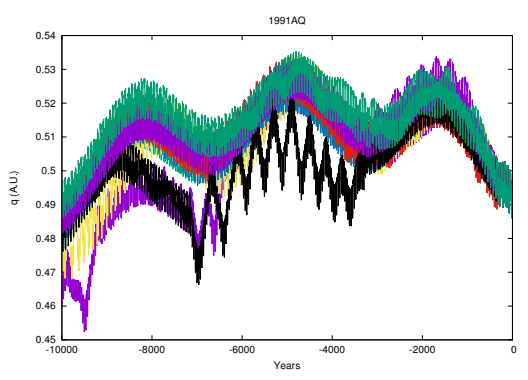

(a)

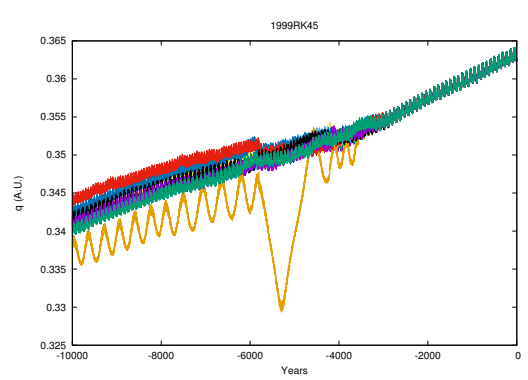

(d)

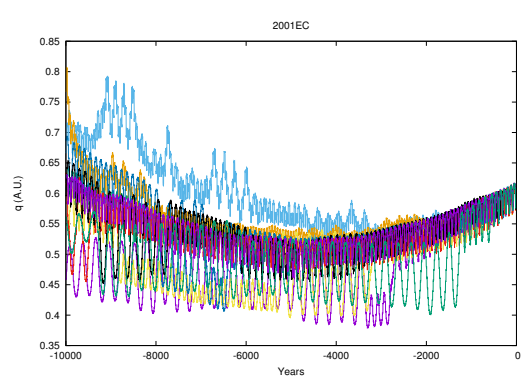

(g)

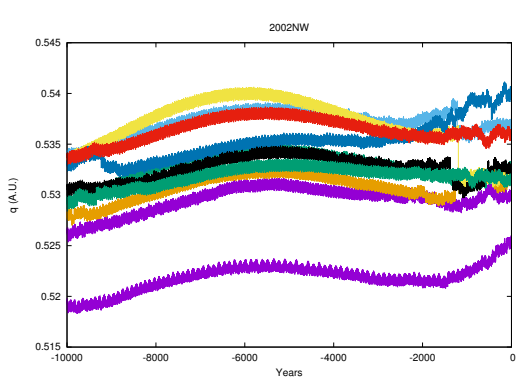

(j)

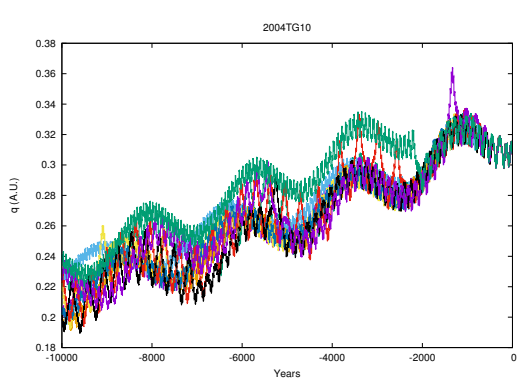

(m)

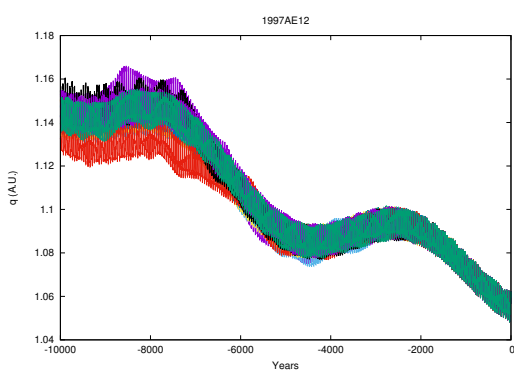

(b)

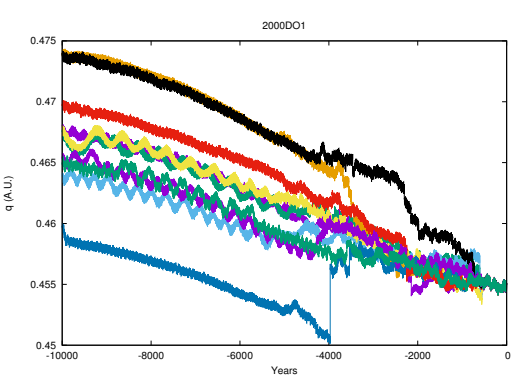

(e)

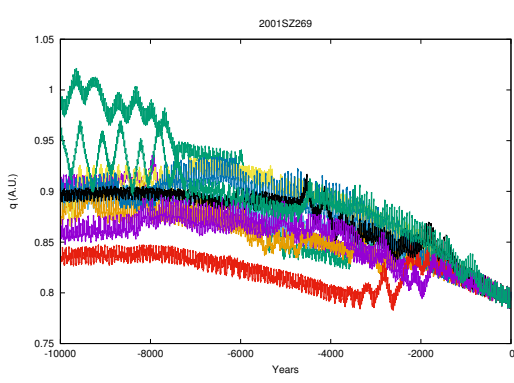

(h)

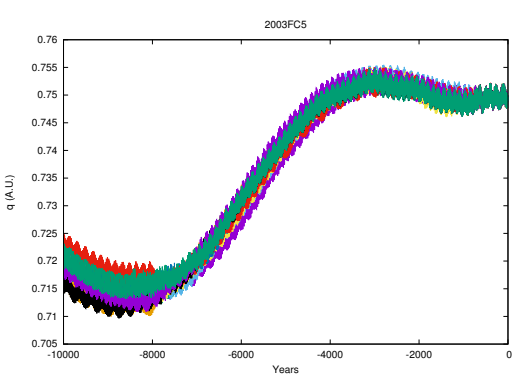

(k)

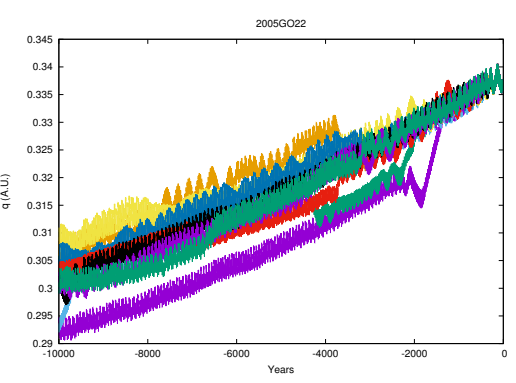

(n)

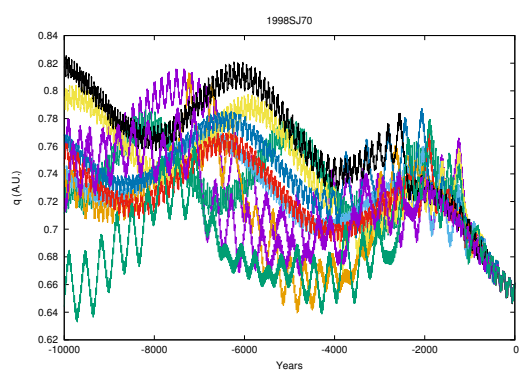

(c)

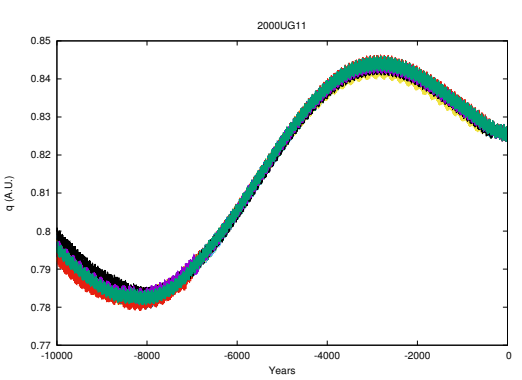

(f)

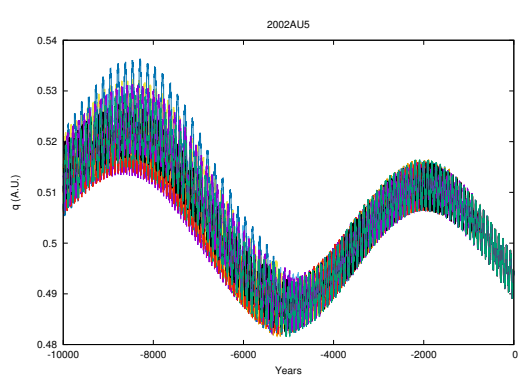

(i)

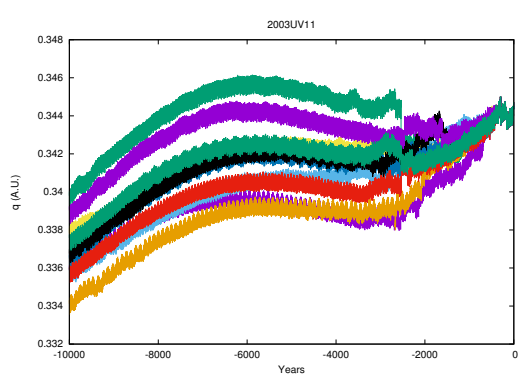

(1)

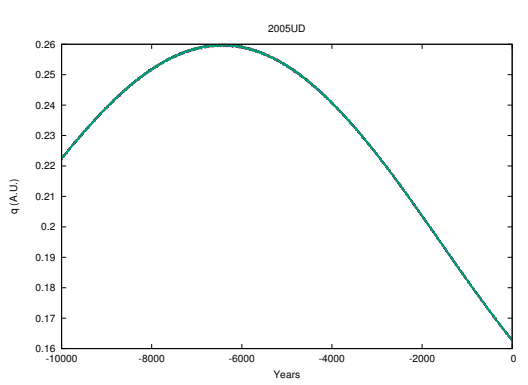

(o)

Fig. D.1. Perihelion $(q)$ evolution of asteroids having physical data (taxonomic class, albedo or/and rotational period). For every asteroid ten clones were integrated $10000 \mathrm{yr}$ backward in time. 


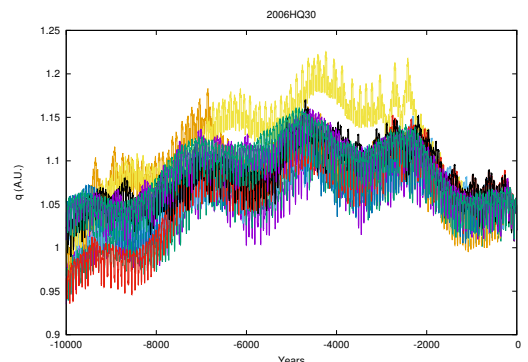

(a)

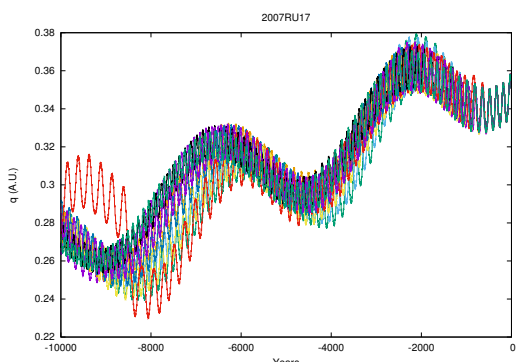

(d)

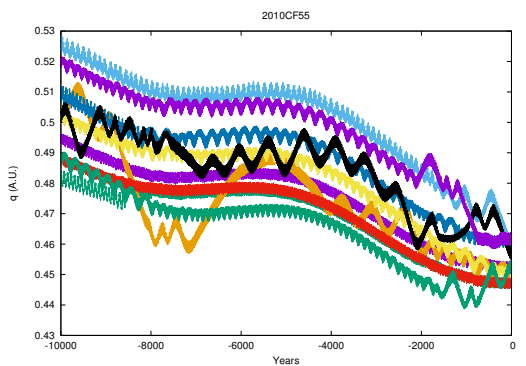

(g)

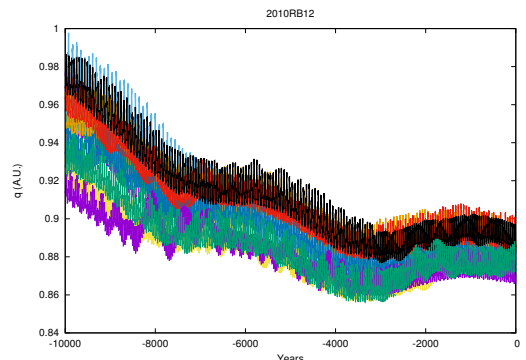

(j)

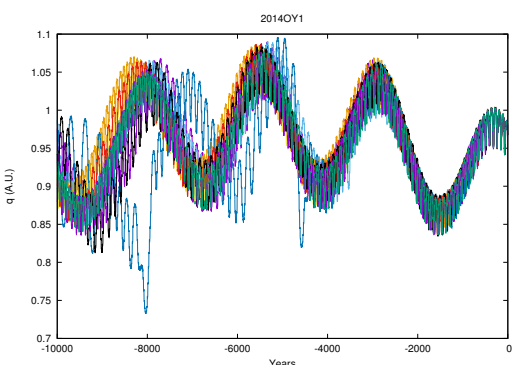

(m)

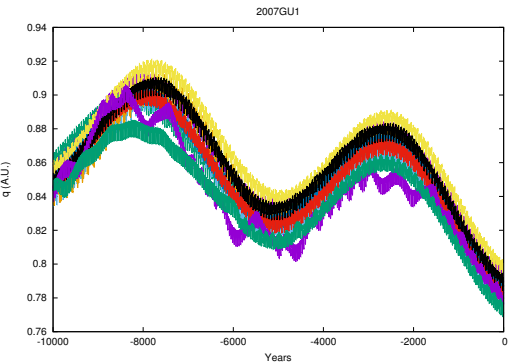

(b)

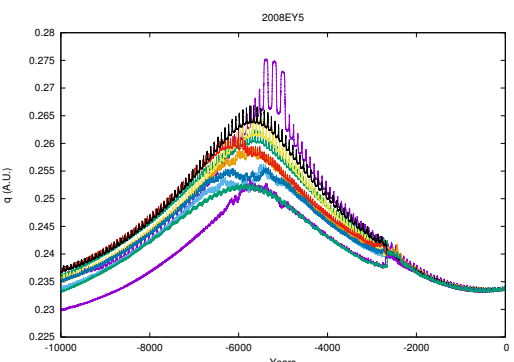

(e)

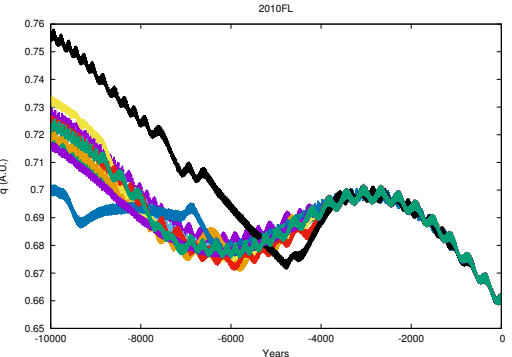

(h)

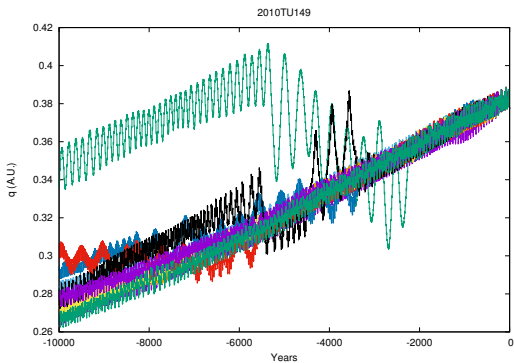

(k)

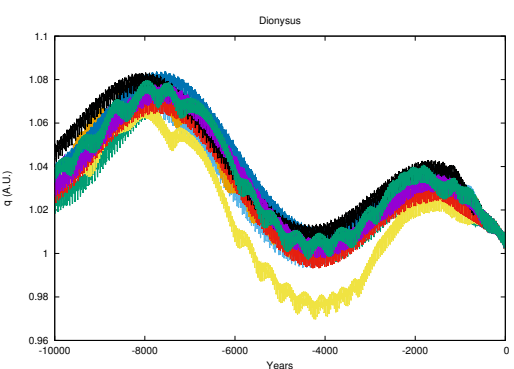

(n)

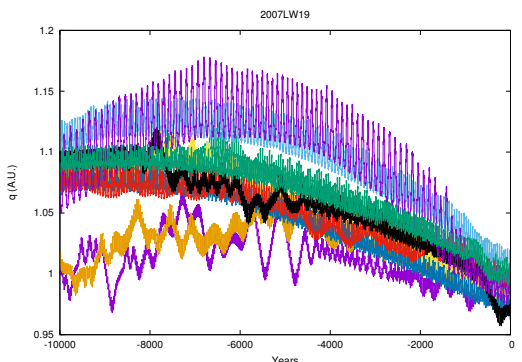

(c)

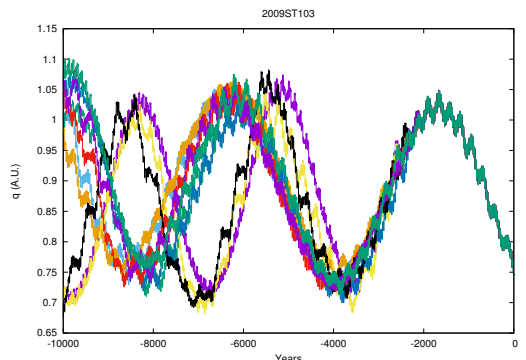

(f)

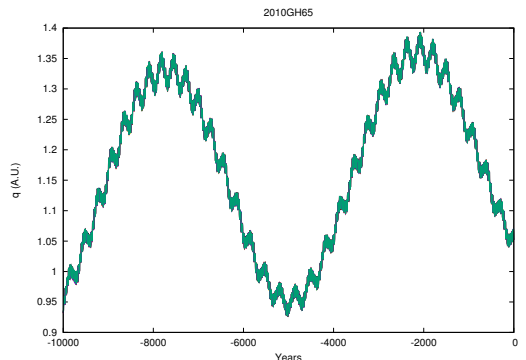

(i)

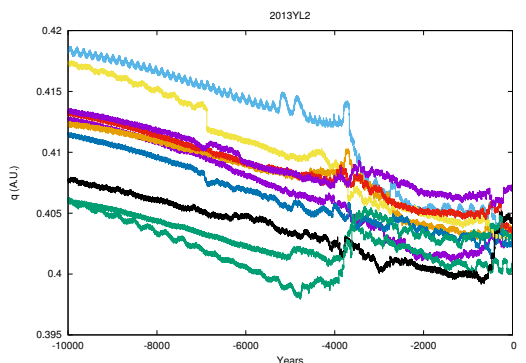

(1)

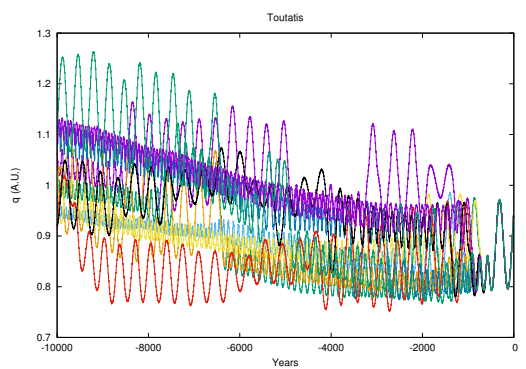

(o)

Fig. D.2. Perihelion $(q)$ evolution of asteroids having physical data (taxonomic class, albedo or/and rotational period). For every asteroid ten clones were integrated $10000 \mathrm{yr}$ backward in time. 
B. A. Dumitru et al:: Association between meteor showers and asteroids using multivariate criteria

Appendix E: D-parameter and Lyapunov time

Table E.1. $D$-parameter and Lyapunov time for all asteroids-meteor showers associations.

\begin{tabular}{|c|c|c|c|c|c|c|c|c|c|c|c|}
\hline Asteroids & $a[\mathrm{au}]$ & $e$ & $\begin{array}{l}T_{\mathrm{L}} \\
\text { [years] }\end{array}$ & $\begin{array}{l}\sigma_{T_{\mathrm{L}}} \\
\text { [years] }\end{array}$ & $\begin{array}{l}\text { Associated } \\
\text { shower }\end{array}$ & $D_{\mathrm{ACS}}$ & $\sigma_{D_{\mathrm{ACS}}}$ & $D_{\mathrm{SH}}$ & $\sigma_{D_{\mathrm{SH}}}$ & $D_{\mathrm{H}}$ & $\sigma_{D_{\mathrm{H}}}$ \\
\hline Adonis & 1.874 & 0.765 & 34 & 1 & ORS & & & 0.155 & $1.5 \mathrm{E}-05$ & 0.161 & $1.5 \mathrm{E}-05$ \\
\hline Dionysus & 2.199 & 0.541 & 52 & 1 & TAH & & & 0.173 & $4.0 \mathrm{E}-07$ & 0.170 & $4.1 \mathrm{E}-07$ \\
\hline Phaethon & 1.271 & 0.890 & 226 & 6 & GEM & 0.017 & $1.9 \mathrm{E}-07$ & 0.032 & $3.0 \mathrm{E}-07$ & 0.036 & $2.8 \mathrm{E}-07$ \\
\hline Toutatis & 2.535 & 0.629 & 59 & 1 & $\mathrm{OCC}$ & & & 0.180 & $9.6 \mathrm{E}-07$ & 0.175 & $9.9 \mathrm{E}-07$ \\
\hline 1991AQ & 2.222 & 0.777 & 94 & 3 & $\mathrm{NCC}$ & 0.038 & 9.1E-08 & 0.132 & $3.3 \mathrm{E}-06$ & 0.138 & $3.2 \mathrm{E}-06$ \\
\hline 1996MQ & 2.391 & 0.579 & 75 & 2 & COR & 0.022 & $3.5 \mathrm{E}-03$ & 0.124 & $1.8 \mathrm{E}-03$ & 0.123 & $1.7 \mathrm{E}-03$ \\
\hline 1997AE12 & 2.369 & 0.554 & 172 & 6 & COR & 0.043 & $1.8 \mathrm{E}-07$ & 0.193 & $9.6 \mathrm{E}-07$ & 0.187 & $1.0 \mathrm{E}-06$ \\
\hline 1997GD32 & 2.103 & 0.599 & 101 & 3 & AVB & & & 0.169 & $2.0 \mathrm{E}-03$ & 0.150 & $1.0 \mathrm{E}-03$ \\
\hline 1998LE & 1.507 & 0.697 & 46 & 1 & SSG & & & 0.111 & 7.3E-03 & 0.111 & 7.3E-03 \\
\hline $1998 \mathrm{SH} 2$ & 2.745 & 0.714 & 172 & 6 & AVB & & & 0.102 & $2.6 \mathrm{E}-06$ & 0.097 & $2.7 \mathrm{E}-06$ \\
\hline 1998SJ70 & 2.238 & 0.705 & 44 & 1 & AVB & & & 0.145 & $2.3 \mathrm{E}-06$ & 0.133 & $2.4 \mathrm{E}-06$ \\
\hline 1998YM4 & 1.477 & 0.720 & 89 & 1 & SCC & & & 0.183 & $2.5 \mathrm{E}-05$ & 0.183 & $2.5 \mathrm{E}-05$ \\
\hline 1999LW1 & 1.438 & 0.682 & 229 & 11 & SSG & & & 0.146 & $9.9 \mathrm{E}-06$ & 0.146 & $9.9 \mathrm{E}-06$ \\
\hline 1999RK33 & 2.479 & 0.583 & 61 & 1 & COR & 0.045 & $1.1 \mathrm{E}-02$ & 0.112 & $6.9 \mathrm{E}-03$ & 0.108 & $5.3 \mathrm{E}-03$ \\
\hline 1999RK45 & 1.598 & 0.773 & 465 & 27 & $\mathrm{ZPE}$ & 0.041 & $1.1 \mathrm{E}-06$ & 0.150 & $1.2 \mathrm{E}-05$ & 0.153 & $1.1 \mathrm{E}-05$ \\
\hline 1999RR28 & 1.879 & 0.653 & 48 & 1 & AND & & & 0.184 & $1.6 \mathrm{E}-07$ & 0.168 & $1.7 \mathrm{E}-07$ \\
\hline 1999VF22 & 1.313 & 0.739 & 39 & 1 & EVI & & & 0.142 & $1.2 \mathrm{E}-06$ & 0.167 & $1.1 \mathrm{E}-06$ \\
\hline 2000DO1 & 1.430 & 0.682 & 38 & 1 & EVI & & & 0.139 & $4.2 \mathrm{E}-07$ & 0.139 & $4.2 \mathrm{E}-07$ \\
\hline 2000UG11 & 1.929 & 0.573 & 85 & 3 & AND & & & 0.183 & $1.5 \mathrm{E}-07$ & 0.176 & $1.4 \mathrm{E}-07$ \\
\hline 2001EC & 2.577 & 0.773 & 34 & 1 & CAP & & & 0.143 & $1.0 \mathrm{E}-03$ & 0.143 & $1.0 \mathrm{E}-03$ \\
\hline 2001QJ96 & 1.592 & 0.797 & 50 & 1 & SMA & 0.038 & 8.3E-06 & 0.169 & 8.3E-06 & 0.182 & $1.6 \mathrm{E}-05$ \\
\hline 2001SZ269 & 2.365 & 0.661 & 54 & 1 & HVI & 0.039 & $4.2 \mathrm{E}-07$ & 0.079 & $3.0 \mathrm{E}-06$ & 0.065 & $3.6 \mathrm{E}-06$ \\
\hline $2001 \mathrm{TA} 2$ & 1.770 & 0.647 & 48 & 1 & AVB & & & 0.155 & 5.3E-07 & 0.132 & 4.7E-07 \\
\hline 2001UX4 & 1.721 & 0.753 & 71 & 2 & NTA & & & 0.159 & $1.2 \mathrm{E}-06$ & 0.169 & $1.1 \mathrm{E}-06$ \\
\hline 2002AU5 & 2.020 & 0.755 & 137 & 4 & $\mathrm{SSG}$ & 0.058 & $9.1 \mathrm{E}-07$ & 0.205 & $1.7 \mathrm{E}-06$ & & \\
\hline $2002 \mathrm{CB} 26$ & 2.012 & 0.733 & 160 & 5 & CAP & & & 0.159 & $1.3 \mathrm{E}-02$ & 0.158 & $1.3 \mathrm{E}-02$ \\
\hline $2002 \mathrm{CN} 15$ & 1.324 & 0.696 & 205 & 6 & EVI & & & 0.178 & $3.9 \mathrm{E}-04$ & 0.181 & $4.5 \mathrm{E}-04$ \\
\hline 2002EL6 & 2.302 & 0.577 & 60 & 1 & TAH & & & 0.188 & $7.8 \mathrm{E}-07$ & 0.188 & $7.8 \mathrm{E}-07$ \\
\hline 2002FU5 & 2.495 & 0.699 & 89 & 2 & AVB & 0.054 & 2.7E-04 & 0.183 & $4.2 \mathrm{E}-04$ & 0.182 & $4.2 \mathrm{E}-04$ \\
\hline 2002GJ8 & 3.235 & 0.681 & 63 & 1 & AUD & & & 0.164 & $2.6 \mathrm{E}-05$ & 0.163 & $2.1 \mathrm{E}-05$ \\
\hline 2002GM2 & 2.198 & 0.808 & 96 & 5 & MKA & 0.058 & $3.0 \mathrm{E}-07$ & 0.194 & $6.6 \mathrm{E}-06$ & & \\
\hline 2002NW & 1.612 & 0.669 & 41 & 1 & CAP & & & 0.134 & $4.0 \mathrm{E}-03$ & 0.133 & $4.0 \mathrm{E}-03$ \\
\hline 2002PX39 & 2.461 & 0.594 & 104 & 3 & COR & 0.046 & 2.7E-03 & 0.104 & 5.9E-04 & 0.104 & $5.9 \mathrm{E}-04$ \\
\hline 2002RC 117 & 2.412 & 0.627 & 81 & 4 & $\mathrm{OCC}$ & & & 0.172 & $5.9 \mathrm{E}-03$ & 0.155 & $3.5 \mathrm{E}-03$ \\
\hline 2003FB5 & 2.500 & 0.794 & 154 & 7 & EVI & 0.021 & $4.5 \mathrm{E}-03$ & 0.154 & $2.6 \mathrm{E}-02$ & 0.155 & $2.6 \mathrm{E}-02$ \\
\hline $2003 F C 5$ & 1.916 & 0.609 & 119 & 5 & AND & & & 0.179 & $4.7 \mathrm{E}-07$ & 0.179 & $4.7 \mathrm{E}-07$ \\
\hline 2003LW1 & 2.121 & 0.508 & 391 & 32 & $\mathrm{TAH}$ & & & 0.188 & $3.0 \mathrm{E}-03$ & 0.177 & $1.8 \mathrm{E}-03$ \\
\hline 2003RE2 & 2.466 & 0.541 & 256 & 12 & $\mathrm{COR}$ & 0.049 & 4.1E-04 & 0.179 & $7.0 \mathrm{E}-04$ & 0.136 & $2.0 \mathrm{E}-04$ \\
\hline 2003UQ25 & 2.551 & 0.682 & 36 & 1 & AND & & & 0.159 & $2.2 \mathrm{E}-03$ & 0.154 & $1.2 \mathrm{E}-03$ \\
\hline \multirow[t]{3}{*}{ 2003UV11 } & 1.453 & 0.763 & 86 & 2 & BTA & & & 0.150 & $4.9 \mathrm{E}-07$ & 0.151 & $4.9 \mathrm{E}-07$ \\
\hline & & & & & NTA & & & 0.170 & $5.2 \mathrm{E}-07$ & 0.171 & $5.2 \mathrm{E}-07$ \\
\hline & & & & & STA & & & 0.087 & $4.0 \mathrm{E}-07$ & 0.087 & $4.0 \mathrm{E}-07$ \\
\hline 2004GB2 & 2.118 & 0.651 & 329 & 21 & AND & & & 0.117 & $3.0 \mathrm{E}-04$ & 0.116 & $2.6 \mathrm{E}-04$ \\
\hline 2004HC39 & 1.814 & 0.501 & 722 & 93 & $\mathrm{TAH}$ & & & 0.191 & $2.0 \mathrm{E}-03$ & 0.184 & $1.5 \mathrm{E}-03$ \\
\hline $2004 \mathrm{MC}$ & 2.437 & 0.592 & 65 & 1 & COR & 0.036 & $1.0 \mathrm{E}-03$ & 0.102 & 4.3E-04 & 0.102 & $4.2 \mathrm{E}-04$ \\
\hline 2004RW10 & 2.352 & 0.593 & 30 & 1 & COR & 0.024 & $7.4 \mathrm{E}-07$ & 0.124 & $1.1 \mathrm{E}-05$ & 0.118 & $1.2 \mathrm{E}-05$ \\
\hline 2004SA20 & 2.403 & 0.710 & 30 & 1 & AVB & & & 0.164 & $2.4 \mathrm{E}-02$ & 0.160 & $1.8 \mathrm{E}-02$ \\
\hline \multirow[t]{2}{*}{ 2004TG10 } & 2.234 & 0.862 & 75 & 1 & NTA & 0.052 & $6.6 \mathrm{E}-07$ & 0.125 & $6.1 \mathrm{E}-06$ & 0.135 & $5.7 \mathrm{E}-06$ \\
\hline & & & & & BTA & & & 0.146 & $5.2 \mathrm{E}-06$ & 0.147 & $5.1 \mathrm{E}-06$ \\
\hline 2004VY14 & 1.961 & 0.650 & & & AVB & & & 0.186 & $3.9 \mathrm{E}-06$ & 0.181 & $4.0 \mathrm{E}-06$ \\
\hline $2005 \mathrm{GO} 22$ & 1.913 & 0.824 & 35 & 1 & APS & & & 0.130 & $6.3 \mathrm{E}-06$ & 0.178 & $4.6 \mathrm{E}-06$ \\
\hline 2005JJ91 & 2.783 & 0.600 & 1721 & 462 & TAH & & & 0.187 & $2.8 \mathrm{E}-03$ & 0.139 & $8.9 \mathrm{E}-04$ \\
\hline 2005MR1 & 2.363 & 0.553 & 167 & 5 & COR & 0.022 & $9.6 \mathrm{E}-05$ & 0.110 & $2.1 \mathrm{E}-04$ & 0.098 & $6.6 \mathrm{E}-05$ \\
\hline $2005 \mathrm{OF} 3$ & 2.387 & 0.585 & 145 & 5 & COR & 0.023 & 8.0E-04 & 0.182 & $3.4 \mathrm{E}-04$ & 0.182 & $3.3 \mathrm{E}-04$ \\
\hline
\end{tabular}

Notes. The columns are: the asteroid name, the semi-major axis and the eccentricity, the Lyapunov time and its error, associated meteor shower and the $D$-parameter for all metrics with the corresponding error. 
Table E.1. continued.

\begin{tabular}{|c|c|c|c|c|c|c|c|c|c|c|c|}
\hline Asteroids & $a[\mathrm{au}]$ & $e$ & $\begin{array}{l}T_{\mathrm{L}} \\
\text { [years] }\end{array}$ & $\begin{array}{l}\sigma_{T_{\mathrm{L}}} \\
\text { [years] }\end{array}$ & $\begin{array}{l}\text { Associated } \\
\text { shower }\end{array}$ & $D_{\text {ACS }}$ & $\sigma_{D_{\mathrm{ACS}}}$ & $D_{\mathrm{SH}}$ & $\sigma_{D_{\mathrm{SH}}}$ & $D_{\mathrm{H}}$ & $\sigma_{D_{\mathrm{H}}}$ \\
\hline 2005RW3 & 2.106 & 0.645 & 79 & 2 & AVB & & & 0.154 & $3.0 \mathrm{E}-03$ & 0.154 & $3.0 \mathrm{E}-03$ \\
\hline 2005 TВ15 & 1.812 & 0.756 & 43 & 1 & STA & & & 0.119 & $8.6 \mathrm{E}-07$ & 0.137 & 8.0E-07 \\
\hline 2005TD49 & 2.684 & 0.626 & 129 & 4 & $\mathrm{OCC}$ & & & 0.163 & $6.0 \mathrm{E}-05$ & 0.163 & $5.7 \mathrm{E}-05$ \\
\hline 2005TE & 1.748 & 0.577 & 45 & 1 & AVB & & & 0.158 & $1.3 \mathrm{E}-02$ & 0.158 & $1.3 \mathrm{E}-02$ \\
\hline 2005UD & 1.275 & 0.872 & 478 & 21 & DSX & & & 0.152 & $9.0 \mathrm{E}-07$ & 0.160 & $9.7 \mathrm{E}-07$ \\
\hline 2006HQ30 & 2.595 & 0.607 & 403 & 31 & TAH & & & 0.191 & $1.1 \mathrm{E}-04$ & 0.186 & 4.3E-05 \\
\hline $2006 \mathrm{JO}$ & 2.379 & 0.667 & 125 & 6 & AVB & & & 0.156 & 4.3E-03 & 0.152 & $2.2 \mathrm{E}-03$ \\
\hline $20060 \mathrm{OC} 5$ & 2.399 & 0.653 & 127 & 3 & $\mathrm{OCC}$ & & & 0.206 & $6.4 \mathrm{E}-07$ & 0.162 & 4.3E-07 \\
\hline 2006PF1 & 2.193 & 0.877 & 199 & 10 & NIA & & & 0.171 & 4.1E-03 & 0.182 & $6.9 \mathrm{E}-03$ \\
\hline 2006UF17 & 2.476 & 0.811 & 109 & 2 & EVI & 0.029 & $3.2 \mathrm{E}-04$ & 0.119 & $1.9 \mathrm{E}-03$ & 0.120 & $1.9 \mathrm{E}-03$ \\
\hline 2006XA3 & 2.361 & 0.624 & 149 & 7 & $\mathrm{OCC}$ & & & 0.199 & $1.0 \mathrm{E}-02$ & 0.181 & $5.6 \mathrm{E}-03$ \\
\hline 2006XG1 & 2.459 & 0.595 & 65 & 2 & PPU & & & 0.137 & 1.9E-06 & 0.136 & $1.9 \mathrm{E}-06$ \\
\hline 2007EJ88 & 2.325 & 0.779 & 33 & 1 & EVI & & & 0.158 & $1.0 \mathrm{E}-02$ & 0.158 & $1.0 \mathrm{E}-02$ \\
\hline 2007GU1 & 2.183 & 0.640 & 224 & 14 & AVB & & & 0.139 & $1.8 \mathrm{E}-03$ & 0.135 & $1.1 \mathrm{E}-03$ \\
\hline 2007LW19 & 2.346 & 0.581 & 56 & 1 & COR & 0.013 & $4.0 \mathrm{E}-03$ & 0.116 & $1.6 \mathrm{E}-02$ & 0.115 & $1.6 \mathrm{E}-02$ \\
\hline 2007RU17 & 2.039 & 0.828 & 50 & 1 & STA & & & 0.095 & $1.1 \mathrm{E}-06$ & 0.095 & $1.1 \mathrm{E}-06$ \\
\hline \multirow[t]{3}{*}{ 2007UL12 } & 1.966 & 0.806 & 62 & 1 & STA & 0.022 & 8.8E-04 & 0.173 & $5.7 \mathrm{E}-04$ & 0.175 & $9.1 \mathrm{E}-04$ \\
\hline & & & & & BTA & & & 0.177 & $9.7 \mathrm{E}-04$ & 0.186 & $1.7 \mathrm{E}-03$ \\
\hline & & & & & ORS & & & 0.185 & $2.4 \mathrm{E}-04$ & 0.185 & $2.4 \mathrm{E}-04$ \\
\hline 2007YP56 & 1.996 & 0.709 & 50 & 1 & SSG & & & 0.168 & 8.0E-03 & 0.165 & 6.7E-03 \\
\hline 2008BO16 & 2.436 & 0.810 & 522 & 43 & CAP & 0.053 & $1.8 \mathrm{E}-03$ & 0.149 & $1.7 \mathrm{E}-03$ & 0.146 & $1.7 \mathrm{E}-03$ \\
\hline 2008CA22 & 2.029 & 0.728 & 59 & 1 & EVI & & & 0.183 & $1.7 \mathrm{E}-03$ & 0.183 & $1.6 \mathrm{E}-03$ \\
\hline 2008EY5 & 0.626 & 0.627 & 84 & 1 & XSA & & & 0.145 & $6.1 \mathrm{E}-07$ & 0.168 & $5.7 \mathrm{E}-07$ \\
\hline 2008RT & 2.484 & 0.568 & 234 & 10 & PPU & & & 0.157 & $1.3 \mathrm{E}-02$ & 0.143 & $1.4 \mathrm{E}-02$ \\
\hline 2008VL14 & 2.198 & 0.821 & 61 & 1 & EVI & & & 0.142 & 4.3E-03 & 0.147 & $6.0 \mathrm{E}-03$ \\
\hline 2008XQ2 & 2.201 & 0.552 & 104 & 3 & PPU & & & 0.162 & 7.1E-06 & 0.162 & 7.1E-06 \\
\hline 2008XU2 & 2.448 & 0.621 & 45 & 1 & $\mathrm{OCC}$ & & & 0.172 & $2.1 \mathrm{E}-03$ & 0.164 & $1.4 \mathrm{E}-03$ \\
\hline 2009FU4 & 2.380 & 0.616 & 68 & 1 & TAH & & & 0.182 & 4.0E-07 & 0.176 & $3.6 \mathrm{E}-07$ \\
\hline 2009HS44 & 2.574 & 0.702 & 92 & 2 & AVB & & & 0.176 & $9.8 \mathrm{E}-04$ & 0.175 & $4.6 \mathrm{E}-04$ \\
\hline 2009QG2 & 2.329 & 0.574 & 43 & 1 & COR & 0.011 & $3.0 \mathrm{E}-03$ & 0.118 & $1.1 \mathrm{E}-03$ & 0.118 & $9.6 \mathrm{E}-04$ \\
\hline 2009SB15 & 2.047 & 0.678 & 56 & 1 & AVB & & & 0.159 & 4.3E-06 & 0.147 & 4.2E-06 \\
\hline 2009ST103 & 2.687 & 0.722 & 389 & 26 & AND & & & 0.135 & $2.1 \mathrm{E}-06$ & 0.135 & $2.1 \mathrm{E}-06$ \\
\hline 2009ST171 & 2.577 & 0.609 & 49 & 1 & $\mathrm{OCC}$ & & & 0.171 & $9.1 \mathrm{E}-03$ & 0.170 & 8.3E-03 \\
\hline 2009SX17 & 2.338 & 0.542 & 241 & 15 & COR & 0.036 & $1.7 \mathrm{E}-04$ & 0.171 & 4.7E-04 & 0.159 & 4.1E-04 \\
\hline 2009TA1 & 2.291 & 0.664 & 99 & 3 & AND & & & 0.166 & $3.4 \mathrm{E}-06$ & 0.165 & $3.4 \mathrm{E}-06$ \\
\hline 2009WJ1 & 2.467 & 0.674 & 43 & 1 & AND & & & 0.190 & $1.2 \mathrm{E}-03$ & 0.187 & $6.8 \mathrm{E}-04$ \\
\hline 2010 CF55 & 1.902 & 0.760 & 56 & 1 & EVI & & & 0.097 & $1.4 \mathrm{E}-03$ & 0.097 & $1.4 \mathrm{E}-03$ \\
\hline 2010FL & 1.916 & 0.655 & 324 & 24 & AVB & & & 0.164 & $9.9 \mathrm{E}-07$ & 0.153 & $1.0 \mathrm{E}-06$ \\
\hline 2010GE35 & 2.225 & 0.617 & 95 & 2 & AVB & & & 0.192 & $3.0 \mathrm{E}-03$ & 0.173 & $1.5 \mathrm{E}-03$ \\
\hline 2010GH65 & 2.705 & 0.611 & 2299 & 819 & TAH & 0.052 & $1.2 \mathrm{E}-06$ & 0.205 & $1.1 \mathrm{E}-06$ & & \\
\hline 2010RB12 & 2.368 & 0.629 & 100 & 2 & $\mathrm{OCC}$ & & & 0.200 & $2.6 \mathrm{E}-03$ & 0.178 & $1.4 \mathrm{E}-03$ \\
\hline 2010RL43 & 2.344 & 0.622 & 36 & 1 & HVI & 0.043 & $2.4 \mathrm{E}-03$ & 0.149 & $5.9 \mathrm{E}-03$ & 0.097 & $3.2 \mathrm{E}-03$ \\
\hline 2010TN55 & 2.302 & 0.789 & 81 & 2 & EVI & & & 0.100 & $6.2 \mathrm{E}-03$ & 0.100 & $6.6 \mathrm{E}-03$ \\
\hline 2010TP55 & 2.349 & 0.670 & 115 & 4 & HVI & 0.049 & $1.6 \mathrm{E}-04$ & 0.144 & $2.0 \mathrm{E}-04$ & 0.141 & $1.6 \mathrm{E}-04$ \\
\hline \multirow[t]{3}{*}{ 2010TU149 } & 2.201 & 0.828 & 59 & 1 & NTA & 0.030 & $1.0 \mathrm{E}-06$ & 0.104 & $1.9 \mathrm{E}-04$ & 0.106 & $1.9 \mathrm{E}-04$ \\
\hline & & & & & BTA & & & 0.123 & 5.3E-06 & 0.134 & 5.2E-06 \\
\hline & & & & & STA & & & 0.075 & $1.4 \mathrm{E}-06$ & 0.078 & $1.9 \mathrm{E}-06$ \\
\hline 2010TV54 & 1.913 & 0.614 & 88 & 3 & AND & & & 0.181 & $3.0 \mathrm{E}-03$ & 0.180 & $2.8 \mathrm{E}-03$ \\
\hline 2010UY6 & 2.662 & 0.613 & 219 & 11 & PPU & & & 0.161 & $2.4 \mathrm{E}-02$ & 0.159 & $2.4 \mathrm{E}-02$ \\
\hline 2010XC11 & 2.516 & 0.850 & 90 & 2 & SCC & & & 0.107 & $1.9 \mathrm{E}-06$ & 0.114 & $1.9 \mathrm{E}-06$ \\
\hline 2011BM45 & 1.920 & 0.804 & 259 & 17 & SSG & 0.050 & 7.9E-04 & 0.133 & 7.4E-04 & 0.143 & 1.1E-03 \\
\hline 2011BW10 & 1.617 & 0.722 & 144 & 4 & SSG & & & 0.094 & $2.4 \mathrm{E}-03$ & 0.094 & $2.4 \mathrm{E}-03$ \\
\hline 2011BY18 & 2.265 & 0.783 & 100 & 2 & SSG & & & 0.170 & 3.9E-04 & 0.170 & 4.2E-04 \\
\hline 2011CG50 & 1.683 & 0.703 & 347 & 18 & CAP & & & 0.160 & $2.0 \mathrm{E}-02$ & 0.157 & $2.0 \mathrm{E}-02$ \\
\hline 2011CT4 & 1.725 & 0.719 & 48 & 1 & SSG & & & 0.104 & $9.5 \mathrm{E}-04$ & 0.105 & $9.8 \mathrm{E}-04$ \\
\hline 2011EF17 & 2.344 & 0.743 & 71 & 1 & AVB & & & 0.191 & $4.5 \mathrm{E}-05$ & 0.166 & $3.3 \mathrm{E}-05$ \\
\hline 2011FZ22 & 2.351 & 0.881 & 144 & 7 & MKA & & & 0.112 & $7.5 \mathrm{E}-03$ & 0.115 & $1.9 \mathrm{E}-02$ \\
\hline 2011GP65 & 2.361 & 0.644 & 63 & 1 & AVB & & & 0.202 & 4.1E-05 & 0.188 & $1.9 \mathrm{E}-05$ \\
\hline 2011HP4 & 2.186 & 0.615 & 121 & 5 & AVB & & & 0.157 & $5.7 \mathrm{E}-03$ & 0.138 & $3.2 \mathrm{E}-03$ \\
\hline
\end{tabular}


B. A. Dumitru et al:: Association between meteor showers and asteroids using multivariate criteria

Table E.1. continued.

\begin{tabular}{|c|c|c|c|c|c|c|c|c|c|c|c|}
\hline Asteroids & $a[\mathrm{au}]$ & $e$ & $\begin{array}{l}T_{\mathrm{L}} \\
\text { [years] }\end{array}$ & $\begin{array}{l}\sigma_{T_{\mathrm{L}}} \\
\text { [years] }\end{array}$ & $\begin{array}{l}\text { Associated } \\
\text { shower }\end{array}$ & $D_{\text {ACS }}$ & $\sigma_{D_{\mathrm{ACS}}}$ & $D_{\mathrm{SH}}$ & $\sigma_{D_{\mathrm{SH}}}$ & $D_{\mathrm{H}}$ & $\sigma_{D_{\mathrm{H}}}$ \\
\hline 2011OL51 & 3.111 & 0.639 & 63 & 2 & OCC & & & 0.209 & $6.2 \mathrm{E}-05$ & 0.171 & $2.2 \mathrm{E}-05$ \\
\hline 2011QE38 & 2.424 & 0.576 & 117 & 3 & COR & 0.031 & $1.2 \mathrm{E}-04$ & 0.090 & 8.0E-05 & 0.087 & $2.1 \mathrm{E}-05$ \\
\hline 2011SV71 & 2.626 & 0.611 & 120 & 3 & $\mathrm{TAH}$ & & & 0.132 & $1.4 \mathrm{E}-06$ & 0.124 & $1.5 \mathrm{E}-06$ \\
\hline 2011TA4 & 3.094 & 0.650 & 158 & 7 & PPU & 0.049 & $5.9 \mathrm{E}-04$ & 0.159 & $1.8 \mathrm{E}-02$ & 0.141 & $2.0 \mathrm{E}-02$ \\
\hline \multirow{3}{*}{$2011 \mathrm{TC} 4$} & 1.492 & 0.720 & 30 & 1 & BTA & & & 0.160 & $3.8 \mathrm{E}-06$ & 0.181 & $3.5 \mathrm{E}-06$ \\
\hline & & & & & NTA & & & 0.145 & 4.3E-06 & 0.154 & 4.1E-06 \\
\hline & & & & & STA & & & 0.178 & 7.5E-05 & 0.186 & 7.2E-05 \\
\hline $2011 \mathrm{TJ}$ & 1.939 & 0.637 & 184 & 9 & AVB & & & 0.110 & $9.2 \mathrm{E}-04$ & 0.105 & $7.0 \mathrm{E}-04$ \\
\hline 2011TX8 & 0.910 & 0.708 & 82 & 1 & BTA & & & 0.171 & $9.0 \mathrm{E}-06$ & 0.190 & $1.3 \mathrm{E}-05$ \\
\hline 2011YY62 & 2.468 & 0.658 & 101 & 2 & $\mathrm{OCC}$ & & & 0.202 & $4.4 \mathrm{E}-03$ & 0.163 & $2.1 \mathrm{E}-03$ \\
\hline 2012BJ14 & 2.064 & 0.743 & 80 & 2 & SSG & 0.030 & 2.3E-02 & 0.184 & $1.8 \mathrm{E}-02$ & & \\
\hline 2012BL14 & 1.723 & 0.653 & 47 & 1 & CAP & & & 0.138 & $5.2 \mathrm{E}-02$ & 0.138 & $5.1 \mathrm{E}-02$ \\
\hline 2012BU61 & 2.521 & 0.780 & 139 & 6 & SSG & & & 0.113 & 2.9E-06 & 0.112 & $2.6 \mathrm{E}-06$ \\
\hline $2012 C C 29$ & 2.436 & 0.775 & 38 & 1 & CAP & & & 0.149 & $1.5 \mathrm{E}-03$ & 0.148 & $1.2 \mathrm{E}-03$ \\
\hline 2012ES10 & 1.887 & 0.757 & 101 & 4 & STA & 0.053 & $2.5 \mathrm{E}-03$ & 0.154 & $3.9 \mathrm{E}-03$ & 0.172 & $4.6 \mathrm{E}-03$ \\
\hline 2012FG & 2.086 & 0.635 & 46 & 1 & AND & & & 0.176 & $9.5 \mathrm{E}-04$ & 0.176 & $9.5 \mathrm{E}-04$ \\
\hline 2012FQ62 & 2.182 & 0.725 & 43 & 1 & AVB & & & 0.185 & $3.3 \mathrm{E}-03$ & 0.158 & $2.8 \mathrm{E}-03$ \\
\hline 2012JU & 2.123 & 0.582 & 147 & 9 & AVB & & & 0.206 & $1.0 \mathrm{E}-03$ & 0.172 & 5.3E-04 \\
\hline \multirow[t]{3}{*}{ 2012KA4 } & 1.100 & 0.780 & 71 & 1 & APS & & & 0.189 & $3.3 \mathrm{E}-05$ & 0.189 & $3.3 \mathrm{E}-05$ \\
\hline & & & & & NIA & & & 0.172 & $2.2 \mathrm{E}-06$ & 0.173 & $2.2 \mathrm{E}-06$ \\
\hline & & & & & SMA & & & 0.105 & $3.4 \mathrm{E}-06$ & 0.116 & $3.1 \mathrm{E}-06$ \\
\hline 2012KZ41 & 2.301 & 0.603 & 47 & 1 & COR & 0.040 & $3.0 \mathrm{E}-03$ & 0.136 & $5.5 \mathrm{E}-03$ & 0.115 & $2.0 \mathrm{E}-03$ \\
\hline 2012LJ & 2.323 & 0.699 & 68 & 1 & AVB & & & 0.141 & 9.9E-04 & 0.138 & $5.2 \mathrm{E}-04$ \\
\hline 2012LT & 2.311 & 0.552 & 46 & 1 & COR & 0.055 & $5.6 \mathrm{E}-04$ & 0.189 & $6.3 \mathrm{E}-04$ & 0.187 & $1.6 \mathrm{E}-04$ \\
\hline 2012TT231 & 1.953 & 0.647 & 71 & 3 & AND & & & 0.151 & $5.1 \mathrm{E}-03$ & 0.142 & $3.5 \mathrm{E}-03$ \\
\hline 2012TT5 & 2.075 & 0.655 & 85 & 2 & AVB & & & 0.189 & $2.4 \mathrm{E}-06$ & 0.188 & $2.4 \mathrm{E}-06$ \\
\hline \multirow[t]{2}{*}{ 2012UR158 } & 2.239 & 0.855 & 358 & 20 & NTA & 0.045 & $1.0 \mathrm{E}-07$ & 0.171 & $1.1 \mathrm{E}-05$ & 0.174 & $1.1 \mathrm{E}-05$ \\
\hline & & & & & BTA & & & 0.161 & $1.2 \mathrm{E}-05$ & 0.161 & $1.2 \mathrm{E}-05$ \\
\hline 2012VB5 & 2.089 & 0.651 & 71 & 2 & AND & & & 0.187 & $2.1 \mathrm{E}-03$ & 0.186 & $1.6 \mathrm{E}-03$ \\
\hline $2012 X M 134$ & 3.319 & 0.700 & 94 & 2 & OCC & & & 0.087 & $5.5 \mathrm{E}-03$ & 0.086 & $4.6 \mathrm{E}-03$ \\
\hline 2013AB65 & 1.812 & 0.750 & 32 & 1 & SSG & & & 0.158 & $5.0 \mathrm{E}-04$ & 0.158 & 5.3E-04 \\
\hline 2013СТ36 & 2.459 & 0.818 & 309 & 17 & ORS & & & 0.139 & 7.3E-07 & 0.146 & 7.3E-07 \\
\hline 2013GL8 & 2.429 & 0.843 & 543 & 50 & STA & & & 0.176 & $5.5 \mathrm{E}-06$ & 0.178 & $5.6 \mathrm{E}-06$ \\
\hline 2013JT17 & 2.422 & 0.567 & 172 & 7 & TAH & & & 0.167 & $3.6 \mathrm{E}-03$ & 0.153 & $1.4 \mathrm{E}-03$ \\
\hline 2013NE19 & 2.140 & 0.829 & 132 & 3 & MKA & & & 0.116 & 7.4E-03 & 0.139 & $1.2 \mathrm{E}-02$ \\
\hline 2013PW31 & 2.341 & 0.525 & 199 & 10 & COR & 0.048 & $6.5 \mathrm{E}-04$ & 0.138 & $1.9 \mathrm{E}-03$ & 0.096 & $6.6 \mathrm{E}-04$ \\
\hline 2013RN21 & 2.425 & 0.560 & 96 & 2 & COR & 0.048 & $3.1 \mathrm{E}-04$ & 0.158 & 4.7E-04 & 0.146 & $1.5 \mathrm{E}-04$ \\
\hline 2013RU9 & 2.490 & 0.585 & 230 & 10 & COR & 0.056 & $6.0 \mathrm{E}-04$ & 0.115 & $3.7 \mathrm{E}-04$ & 0.111 & $1.1 \mathrm{E}-04$ \\
\hline 2013WX44 & 2.819 & 0.664 & 140 & 5 & OCC & & & 0.131 & 4.3E-03 & 0.126 & $2.7 \mathrm{E}-03$ \\
\hline 2013XT21 & 2.478 & 0.653 & 58 & 1 & $\mathrm{OCC}$ & & & 0.178 & $5.7 \mathrm{E}-03$ & 0.143 & $2.8 \mathrm{E}-03$ \\
\hline 2013YL2 & 1.185 & 0.660 & 45 & 1 & $\mathrm{NCC}$ & & & 0.176 & $1.1 \mathrm{E}-03$ & 0.176 & $1.1 \mathrm{E}-03$ \\
\hline 2014HT178 & 2.393 & 0.639 & 544 & 60 & AVB & & & 0.152 & $5.4 \mathrm{E}-02$ & 0.119 & $2.7 \mathrm{E}-02$ \\
\hline \multirow[t]{2}{*}{ 2014HU2 } & 2.338 & 0.672 & 121 & 4 & HVI & 0.024 & $5.2 \mathrm{E}-03$ & 0.123 & $2.1 \mathrm{E}-03$ & 0.122 & $1.5 \mathrm{E}-03$ \\
\hline & & & & & AVB & & & 0.122 & 2.0E-03 & 0.120 & $1.3 \mathrm{E}-03$ \\
\hline 2014JH15 & 2.316 & 0.624 & 64 & 1 & HVI & 0.038 & $3.1 \mathrm{E}-03$ & 0.149 & 7.9E-03 & 0.110 & $3.9 \mathrm{E}-03$ \\
\hline 2014KH39 & 2.343 & 0.593 & 102 & 4 & COR & 0.054 & $3.4 \mathrm{E}-06$ & 0.073 & $1.7 \mathrm{E}-05$ & 0.062 & $6.0 \mathrm{E}-06$ \\
\hline 2014MC6 & 2.215 & 0.630 & 159 & 4 & AVB & & & 0.190 & $3.8 \mathrm{E}-05$ & 0.180 & $2.1 \mathrm{E}-05$ \\
\hline \multirow[t]{2}{*}{ 2014NK52 } & 2.204 & 0.839 & 200 & 8 & NTA & 0.028 & $1.7 \mathrm{E}-03$ & 0.144 & $6.3 \mathrm{E}-04$ & 0.144 & $6.3 \mathrm{E}-04$ \\
\hline & & & & & BTA & & & 0.148 & 7.2E-04 & 0.151 & $9.4 \mathrm{E}-04$ \\
\hline 2014OM207 & 2.425 & 0.583 & 45 & 1 & COR & 0.028 & 4.3E-03 & 0.066 & $1.7 \mathrm{E}-03$ & 0.065 & 7.0E-04 \\
\hline 2014006 & 2.202 & 0.679 & 36 & 1 & CAP & & & 0.199 & $2.7 \mathrm{E}-05$ & 0.183 & $1.8 \mathrm{E}-05$ \\
\hline 2014OY1 & 2.578 & 0.625 & 72 & 1 & TAH & & & 0.175 & $1.1 \mathrm{E}-05$ & 0.175 & $1.1 \mathrm{E}-05$ \\
\hline 2014XD32 & 2.060 & 0.653 & 55 & 1 & AVB & & & 0.151 & $2.0 \mathrm{E}-02$ & 0.150 & $1.5 \mathrm{E}-02$ \\
\hline 2014XE32 & 2.492 & 0.651 & 61 & 1 & $\mathrm{OCC}$ & & & 0.175 & $1.8 \mathrm{E}-02$ & 0.144 & 8.7E-03 \\
\hline 2014XL6 & 2.534 & 0.617 & 51 & 1 & OCC & & & 0.173 & $5.3 \mathrm{E}-03$ & 0.172 & $5.0 \mathrm{E}-03$ \\
\hline 2015 BA513 & 1.723 & 0.695 & 53 & 1 & SSG & & & 0.171 & $1.7 \mathrm{E}-02$ & 0.171 & $1.7 \mathrm{E}-02$ \\
\hline 2015CE1 & 1.591 & 0.660 & 97 & 2 & CAP & & & 0.124 & $2.4 \mathrm{E}-03$ & 0.123 & 2.3E-03 \\
\hline 2015CP & 2.409 & 0.796 & 151 & 6 & CAP & & & 0.109 & $1.6 \mathrm{E}-02$ & 0.105 & $1.6 \mathrm{E}-02$ \\
\hline 2015DA54 & 2.614 & 0.780 & 87 & 2 & CAP & 0.029 & 8.9E-05 & 0.058 & 7.3E-06 & 0.058 & $6.5 \mathrm{E}-06$ \\
\hline
\end{tabular}


Table E.1. continued.

\begin{tabular}{|c|c|c|c|c|c|c|c|c|c|c|c|}
\hline Asteroids & $a[\mathrm{au}]$ & $e$ & $\begin{array}{l}T_{\mathrm{L}} \\
\text { [years] }\end{array}$ & $\begin{array}{l}\sigma_{T_{\mathrm{L}}} \\
\text { [years] }\end{array}$ & $\begin{array}{l}\text { Associated } \\
\text { shower }\end{array}$ & $D_{\text {ACS }}$ & $\sigma_{D_{\mathrm{ACS}}}$ & $D_{\mathrm{SH}}$ & $\sigma_{D_{\mathrm{SH}}}$ & $D_{\mathrm{H}}$ & $\sigma_{D_{\mathrm{H}}}$ \\
\hline 2015DE54 & 2.144 & 0.801 & 182 & 7 & CAP & & & 0.167 & $1.8 \mathrm{E}-02$ & 0.166 & $2.1 \mathrm{E}-02$ \\
\hline 2015ER & 2.317 & 0.816 & 73 & 2 & EVI & & & 0.136 & $1.5 \mathrm{E}-03$ & 0.138 & $1.9 \mathrm{E}-03$ \\
\hline 2015FD35 & 1.517 & 0.672 & 81 & 1 & EVI & & & 0.172 & $1.5 \mathrm{E}-06$ & 0.172 & $1.5 \mathrm{E}-06$ \\
\hline 2015FQ & 2.245 & 0.715 & 58 & 1 & AVB & & & 0.193 & $7.4 \mathrm{E}-06$ & 0.179 & $4.5 \mathrm{E}-06$ \\
\hline 2015FQ117 & 0.861 & 0.621 & 28 & 1 & XSA & & & 0.150 & $1.3 \mathrm{E}-05$ & 0.160 & $1.3 \mathrm{E}-05$ \\
\hline 2015GJ13 & 2.468 & 0.713 & 47 & 1 & AVB & & & 0.181 & $2.2 \mathrm{E}-03$ & 0.179 & $1.1 \mathrm{E}-03$ \\
\hline $2015 \mathrm{KK}$ & 2.555 & 0.786 & 217 & 9 & OER & & & 0.125 & $3.3 \mathrm{E}-03$ & 0.124 & $3.0 \mathrm{E}-03$ \\
\hline 2015MN11 & 2.041 & 0.705 & 44 & 1 & SSG & & & 0.184 & $4.9 \mathrm{E}-03$ & 0.178 & $4.0 \mathrm{E}-03$ \\
\hline $2015 \mathrm{NJ} 3$ & 1.688 & 0.671 & 214 & 7 & CAP & & & 0.175 & $4.0 \mathrm{E}-06$ & 0.174 & $3.9 \mathrm{E}-06$ \\
\hline 2015РМ307 & 2.199 & 0.862 & 88 & 1 & NIA & & & 0.093 & $1.8 \mathrm{E}-02$ & 0.144 & $3.4 \mathrm{E}-02$ \\
\hline 2015PU228 & 2.282 & 0.790 & 593 & 73 & $\mathrm{NCC}$ & & & 0.147 & $1.0 \mathrm{E}-04$ & 0.151 & $1.1 \mathrm{E}-04$ \\
\hline 2015TL143 & 2.584 & 0.604 & 66 & 1 & $\mathrm{OCC}$ & & & 0.187 & $1.1 \mathrm{E}-04$ & 0.184 & 8.7E-05 \\
\hline \multirow[t]{2}{*}{ 2015TX24 } & 2.269 & 0.872 & 77 & 1 & BTA & & & 0.177 & $6.0 \mathrm{E}-04$ & 0.182 & $1.3 \mathrm{E}-03$ \\
\hline & & & & & STA & & & 0.164 & $1.0 \mathrm{E}-03$ & 0.181 & 2.3E-03 \\
\hline 2015VH66 & 2.279 & 0.848 & 181 & 5 & BTA & & & 0.180 & $1.3 \mathrm{E}-03$ & 0.181 & $1.3 \mathrm{E}-03$ \\
\hline 2015VR65 & 2.593 & 0.617 & 161 & 5 & PPU & & & 0.122 & $1.8 \mathrm{E}-03$ & 0.122 & $1.6 \mathrm{E}-03$ \\
\hline 2016BN14 & 2.368 & 0.755 & 166 & 7 & CAP & & & 0.057 & $2.2 \mathrm{E}-03$ & 0.057 & $2.1 \mathrm{E}-03$ \\
\hline 2016BP14 & 2.854 & 0.767 & 50 & 1 & CAP & & & 0.140 & $1.1 \mathrm{E}-03$ & 0.130 & $7.0 \mathrm{E}-04$ \\
\hline 2016BV14 & 2.362 & 0.711 & 45 & 1 & CAP & & & 0.139 & $5.9 \mathrm{E}-04$ & 0.124 & $4.0 \mathrm{E}-04$ \\
\hline 2016CA136 & 2.031 & 0.782 & 44 & 1 & SSG & & & 0.121 & $4.2 \mathrm{E}-04$ & 0.121 & $4.2 \mathrm{E}-04$ \\
\hline 2016CL137 & 1.916 & 0.711 & 19 & 1 & CAP & & & 0.178 & $1.0 \mathrm{E}-03$ & 0.178 & $9.1 \mathrm{E}-04$ \\
\hline \multirow[t]{2}{*}{ 2016CM246 } & 1.948 & 0.776 & 710 & 74 & SSG & 0.026 & 7.9E-03 & 0.110 & $2.9 \mathrm{E}-03$ & 0.110 & $3.6 \mathrm{E}-03$ \\
\hline & & & & & STA & 0.027 & $5.0 \mathrm{E}-03$ & 0.153 & 7.3E-03 & 0.166 & $9.5 \mathrm{E}-03$ \\
\hline 2016CW264 & 2.020 & 0.765 & 178 & 11 & CAP & & & 0.133 & 8.0E-03 & 0.129 & $8.2 \mathrm{E}-03$ \\
\hline 2016ES155 & 1.431 & 0.655 & 76 & 1 & EVI & & & 0.185 & $2.0 \mathrm{E}-03$ & 0.185 & $2.0 \mathrm{E}-03$ \\
\hline 2016EV28 & 1.946 & 0.770 & 72 & 1 & EVI & & & 0.145 & $1.0 \mathrm{E}-04$ & 0.145 & $1.1 \mathrm{E}-04$ \\
\hline 2016FC14 & 2.232 & 0.659 & 72 & 1 & AND & & & 0.136 & 7.2E-03 & 0.136 & $7.2 \mathrm{E}-03$ \\
\hline 2016HN3 & 2.150 & 0.554 & 86 & 2 & TAH & & & 0.163 & 4.9E-05 & 0.162 & 4.5E-05 \\
\hline 2016JD18 & 2.794 & 0.689 & 46 & 1 & AVB & & & 0.164 & $7.9 \mathrm{E}-05$ & 0.132 & $3.5 \mathrm{E}-05$ \\
\hline 2016JS5 & 2.105 & 0.627 & 157 & 6 & AVB & & & 0.191 & $1.9 \mathrm{E}-04$ & 0.188 & $1.2 \mathrm{E}-04$ \\
\hline 2016LE10 & 2.420 & 0.619 & 49 & 1 & COR & 0.054 & $1.6 \mathrm{E}-03$ & 0.199 & $1.7 \mathrm{E}-03$ & 0.188 & $6.7 \mathrm{E}-04$ \\
\hline 2016LW9 & 1.116 & 0.734 & 151 & 4 & SMA & & & 0.135 & $2.5 \mathrm{E}-02$ & 0.140 & $3.0 \mathrm{E}-02$ \\
\hline 2016LZ8 & 2.256 & 0.530 & 115 & 4 & COR & 0.052 & $1.3 \mathrm{E}-03$ & 0.108 & $1.9 \mathrm{E}-03$ & 0.094 & $6.2 \mathrm{E}-04$ \\
\hline $2016 \mathrm{MS}$ & 2.454 & 0.555 & 102 & 3 & COR & 0.041 & $5.7 \mathrm{E}-04$ & 0.145 & $7.9 \mathrm{E}-04$ & 0.120 & $2.1 \mathrm{E}-04$ \\
\hline 2016NG22 & 1.885 & 0.697 & 194 & 5 & SSG & & & 0.177 & $1.3 \mathrm{E}-02$ & 0.175 & $1.2 \mathrm{E}-02$ \\
\hline \multirow[t]{2}{*}{ 2016NO16 } & 2.458 & 0.574 & 75 & 1 & AUD & & & 0.164 & $2.6 \mathrm{E}-05$ & 0.160 & $1.4 \mathrm{E}-05$ \\
\hline & & & & & $\mathrm{KCG}$ & & & 0.179 & $3.1 \mathrm{E}-05$ & 0.173 & $1.6 \mathrm{E}-05$ \\
\hline 2016PD40 & 2.454 & 0.537 & 132 & 4 & COR & 0.050 & $9.3 \mathrm{E}-04$ & 0.192 & $1.7 \mathrm{E}-03$ & 0.149 & $6.1 \mathrm{E}-04$ \\
\hline 2016PN38 & 2.456 & 0.621 & 54 & 1 & OCC & & & 0.186 & $3.5 \mathrm{E}-05$ & 0.179 & $2.3 \mathrm{E}-05$ \\
\hline 2016QY1 & 2.282 & 0.600 & 83 & 2 & COR & 0.037 & $2.8 \mathrm{E}-03$ & 0.160 & $3.5 \mathrm{E}-03$ & 0.142 & $1.3 \mathrm{E}-03$ \\
\hline 2016RO40 & 2.293 & 0.649 & 74 & 1 & AVB & & & 0.176 & $4.3 \mathrm{E}-03$ & 0.170 & $2.1 \mathrm{E}-03$ \\
\hline 2016RX19 & 2.700 & 0.603 & 552 & 54 & BHY & & & 0.187 & 4.1E-04 & 0.162 & $1.5 \mathrm{E}-04$ \\
\hline 2016RZ40 & 2.807 & 0.612 & 185 & 8 & OCC & & & 0.200 & $4.5 \mathrm{E}-03$ & 0.178 & $5.0 \mathrm{E}-03$ \\
\hline 2016SF & 2.131 & 0.608 & 83 & 2 & AVB & & & 0.178 & $7.1 \mathrm{E}-03$ & 0.163 & $3.9 \mathrm{E}-03$ \\
\hline 2016TJ18 & 2.404 & 0.586 & 100 & 3 & PPU & & & 0.116 & $2.9 \mathrm{E}-05$ & 0.116 & $2.9 \mathrm{E}-05$ \\
\hline 2016TP18 & 1.093 & 0.686 & 49 & 1 & BTA & & & 0.184 & $2.8 \mathrm{E}-03$ & 0.185 & $3.0 \mathrm{E}-03$ \\
\hline 2016TW18 & 1.973 & 0.626 & 171 & 7 & AND & & & 0.156 & $6.7 \mathrm{E}-04$ & 0.156 & $6.2 \mathrm{E}-04$ \\
\hline 2016UP36 & 2.536 & 0.692 & 100 & 3 & AND & & & 0.168 & $1.2 \mathrm{E}-03$ & 0.167 & $7.7 \mathrm{E}-04$ \\
\hline 2016 VK & 1.775 & 0.778 & 38 & 1 & NTA & & & 0.176 & $2.6 \mathrm{E}-03$ & 0.179 & $3.8 \mathrm{E}-03$ \\
\hline 2016VQ & 2.503 & 0.661 & 87 & 2 & AND & & & 0.194 & $3.3 \mathrm{E}-03$ & 0.181 & $1.5 \mathrm{E}-03$ \\
\hline 2016WN48 & 2.743 & 0.641 & 46 & 1 & OCC & & & 0.175 & 4.3E-03 & 0.175 & 4.3E-03 \\
\hline
\end{tabular}

